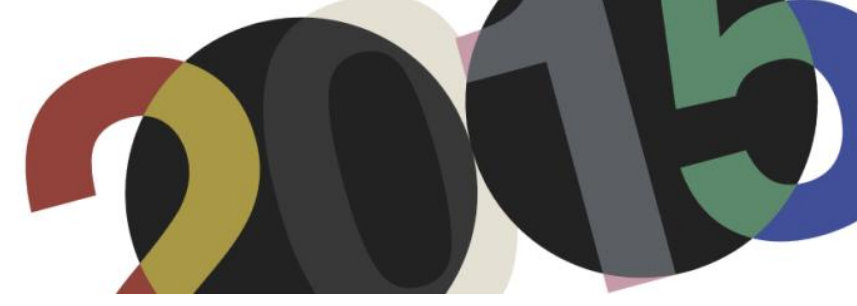

DOI: http://dx.doi.org/10.4995/LC2015.2015.821

\title{
Visions "humaines" ou "infernales": les moyens de transport et la perception de la ville chez Le Corbusier
}

\author{
S. Talenti
}

Université de Salerne, Italie

\begin{abstract}
Résumé: Les représentations des villes depuis la mer ont eu, à partir du XVe siècle, un grand succès dans l'iconographie urbaine des voyageurs. Depuis 1911, Le Corbusier a pris l'habitude d'esquisser dans ses carnets de croquis les paysages urbains découverts au cours de son voyage en Orient. Villes, remparts ou monuments significatifs sont souvent représentés depuis la mer ou les fleuves, car l'arrivée en bateau autorise une perception progressive et globale du site. Suite à son séjour en Amérique du sud en 1929, l'architecte exprime ouvertement son appréciation pour la perspective obtenue depuis le bateau au milieu des baies, car ce point de vue lui permet de contextualiser immédiatement ses propositions d'aménagement urbain. De simple connaissance des villes, cette pratique iconographique devient, chez Le Corbusier, un instrument d'élaboration du projet. Mais la vue horizontale est bientôt accompagnée du procédé de survol des centres urbains pour appréhender la grandeur du paysage naturel et construit. À travers l'analyse des nombreuses images - dessins, croquis, photos - élaborées par Le Corbusier à partir des différents moyens de transport, ainsi que des commentaires accompagnant ces documents iconographiques et de quelques textes publiés, on essayera de mieux comprendre le rapport entre l'échelle de ces visions panoramiques et la nouvelle approche territoriale mise au point par Le Corbusier à partir des années Trente.
\end{abstract}

\begin{abstract}
The representations of cities viewed from the sea have had, since the fifteenth century, a great success in urban iconography. From 1911, Le Corbusier began drawing urban landscapes in his sketchbooks, which he discovered during his trip to the Orient. Cities, fortifications or significant monuments are often depicted from the sea or rivers, because the arrival by boat allows a gradual and overall perception of the site. After his stay in South America in 1929, the architect openly expressed his appreciation for the panoramic view obtained from the boat in the middle of the bay. This point of view allows him to contextualize his urban development proposals immediately. This iconographic practice is no longer a simple function of knowledge, but it has become an instrument for realising the project. However, the horizontal view was soon accompanied by the process of flying over urban centres to understand the magnitude of the man-made and natural landscape. Through the analysis of the many images - drawings, sketches, photos - developed by Le Corbusier using different means of transport, of comments accompanying these iconographic documents and of some published texts, the paper aims to better understand the relationship between the scale of these panoramic visions and the new territorial approach developed by Le Corbusier from the Thirties onwards.
\end{abstract}

Mots clés: moyens de transport; avion; bateau.

Keywords: means of transport; airplane; boat.

\section{Introduction}

“Suddenly... we have been endowed with THE BIRD'S EYE VIEW".

Les représentations des villes depuis la mer ont eu, à partir du XVe siècle, un grand succès dans l'iconographie urbaine des voyageurs. L'approche graduelle de la côte, autorisée par le transport maritime, devient l'occasion pour de nombreux architectes, de découvrir lentement les agglomérations, mais surtout de saisir leur insertion dans le contexte du paysage. Depuis 1911, Le Corbusier a pris l'habitude d'esquisser dans ses carnets de croquis les paysages urbains découverts au cours de son voyage en Orient. Villes, remparts ou monuments significatifs

\footnotetext{
${ }^{1}$ Le Corbusier: Aircraft. London: The Studio, 1935, p. 6.
} 
sont souvent représentés depuis la mer ou les fleuves, car l'arrivée en bateau autorise une perception progressive et globale du site. Suite à son séjour en Amérique du sud en 1929, l'architecte exprime ouvertement son appréciation pour la perspective obtenue depuis le bateau au milieu des baies, car ce point de vue lui permet de contextualiser immédiatement ses propositions d'aménagement urbain. De simple connaissance des villes, cette pratique iconographique devient, chez Le Corbusier, un instrument d'élaboration du projet. Mais la vue horizontale est bientôt accompagnée du procédé de survol des centres urbains pour appréhender la grandeur du

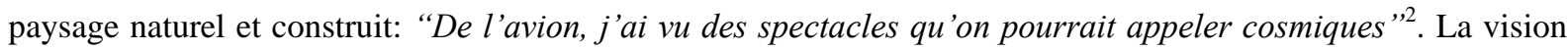
aérienne serait, à son avis, la vue que tout concepteur de ville devrait privilégier afin de mieux comprendre le territoire et ses caractères. En particulier, lors de la conception du Plan Obus pour Alger, la vue d'en haut aurait influencé, selon lui, l'abandon de toute grille orthogonale ${ }^{3}$. Dans la capitale algérienne, où il est invité en 1931, Le Corbusier va tester les différentes façons de voir et de représenter la ville: la vision frontale obtenue depuis le paquebot qui lui permet de mettre l'accent sur l'horizontalité du front de mer; la vue plongeante qui autorise la saisie de la dimension territoriale et enfin celle propre au promeneur qui découvre, en flânant et en pénétrant dans le dédale de la Casbah, le véritable cœur de l'agglomération et sa complexité urbaine. Il s'agit de trois différentes appréhensions de l'espace construit, complémentaires mais basées sur une même saisie relativement lente des données. Le Corbusier est désormais convaincu que seul le paquebot, l'avion et le "pied du marcheur sur la route" peuvent permettre d'apprécier le "spectacle", en produisant des "visions humaines" et globales, contrairement à celles "inhumaines et infernales" engendrées par les trains et les voitures ${ }^{4}$. À travers l'analyse des nombreuses images - dessins, croquis, photos - élaborées par Le Corbusier à partir des différents moyens de transport, ainsi que des commentaires accompagnant ces documents iconographiques et de quelques textes publiés, on essayera de mieux comprendre le rapport entre l'échelle de ces visions panoramiques et la nouvelle approche territoriale mise au point par Le Corbusier à partir des années Trente. Ceci permettra de vérifier jusqu'à quel point les représentations effectuées depuis la mer ou les vues aériennes ne sont pas seulement un moyen de connaissance, mais aussi un outil très important dans la conception de projets d'architecture et d'aménagements urbains qui jaillissent comme des véritables révélations. Les résultats de cette étude ne prétendent pas être exhaustifs, la recherche pouvant encore s'enrichir, un jour, grâce au réexamen de l'œuvre écrite de Le Corbusier ainsi que de sa correspondance privée.

\section{Vision frontale et panoramique}

Pendant le voyage en Orient en 1911, Le Corbusier commence à changer l'orientation de ses carnets de croquis, afin de mieux saisir l'horizontalité des paysages urbains. "Nous sommes venus par la mer, classiquement, pour voir se dérouler les choses". . Les premières impressions depuis le bateau se traduisent en skylines et panoramas, à même de représenter au mieux la spécificité de chaque ville. Nombreux sont les dessins qui, par quelques traits, fixent sur le papier les caractères des grandes agglomérations comme Istanbul, mais aussi de réalités urbaines plus modestes, comme la ville hongroise d'Esztergom dessinée depuis le Danube ${ }^{6}$. Le jeune architecte utilise aussi l'appareil photo pour prendre ses premiers clichés panoramiques depuis l'embarcation: des

\footnotetext{
${ }^{2}$ Le Corbusier: Précisions sur un état présent de l'architecture et de l'urbanisme. Paris: G. Crès et Cie, 1930, p. 4. Cf. Lemonnier, Aurélien: "Des yeux qui accusent: Le Corbusier et la ville vue d'avion". In Lampe Angela (Dir.): Vues d'en haut. Metz: Centre Pompidou, 2013, pp. 258-261.

${ }^{3}$ Cf. Bonillo, Jean-Lucien (Dir.): Le Corbusier. Visions d'Alger. Paris: Editions de la Villette, 2012.

${ }^{4}$ Le Corbusier: Précisions... op. cit., p. 8.

${ }^{5}$ Le Corbusier: Le voyage d'Orient. Marseille: Parenthèses, 1987, p. 67.

${ }^{6}$ De la ville hongroise d'Esztergom dessinée depuis le Danube, Le Corbusier avait également fait une photo en juin 1911: cf. Le Corbusier: Il viaggio d'Oriente (Le voyage d'Orient). Faenza: Faenza Editrice, 1974, p.164 ainsi que Mazza, Barbara: Le Corbusier e la fotografia. La vérité blanche. Firenze: Firenze University Press, 2002, fig. 29.
} 
photosqui visent à saisir les points forts des villes des Balkans ou de quelques citadelles fortifiées, par l'enregistrement de leurs silhouettes.

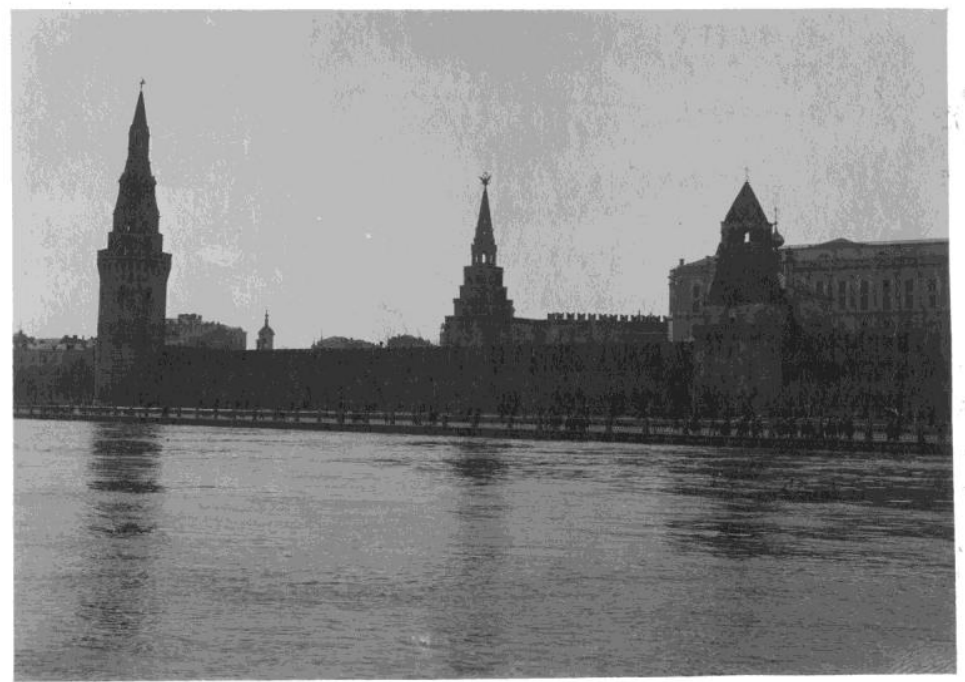

1. La forteresse de Smederevo. Photographie 1911. FLC, L5-1-124.

La forteresse du XVe siècle de la ville serbe de Smederevo est saisie depuis le Danube à travers une image suggestive et séduisante où l'enceinte fortifiée se détache à l'horizon en soulignant l'étendue et l'ampleur de la citadelle $^{7}$. Même les profils noirs et bidimensionnels des villes sont captés dans le but de cristalliser la première image d'un site, selon une approche visuelle qui deviendra une démarche cognitive amplement utilisée par Le Corbusier.

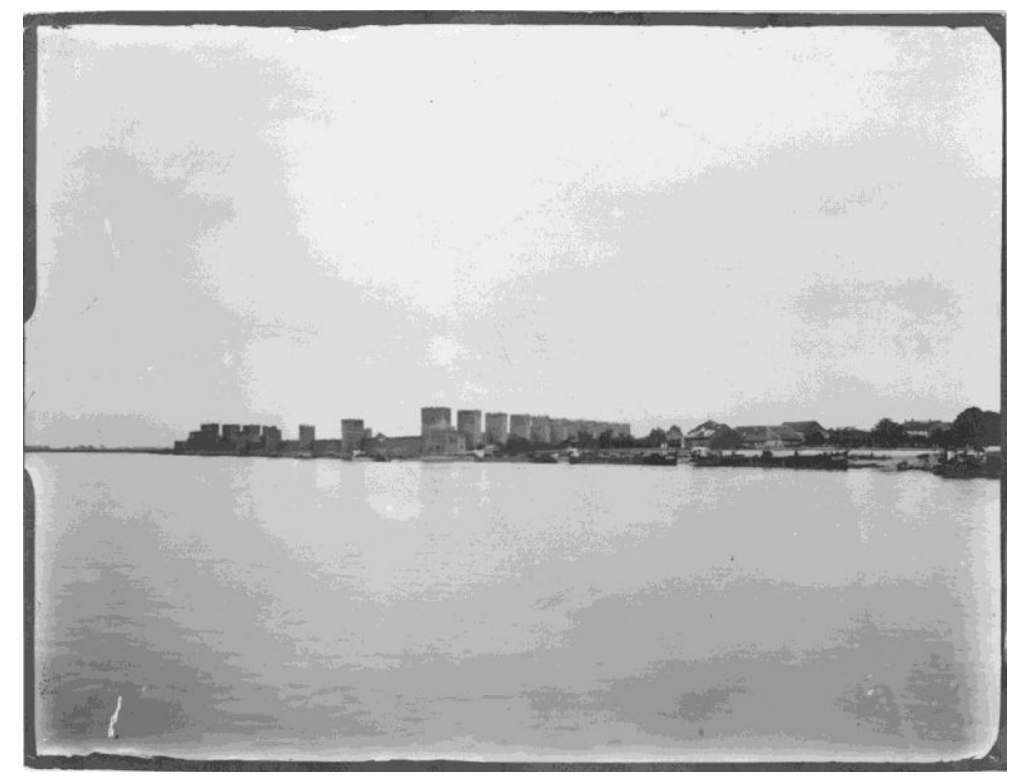

2. Photo prise lors du voyage dans les Balkans en 1911. FLC, L4-20-170.

Le medium photographique ne remplacera toutefois pas le dessin, en particulier dans la saisie des sensations, comme Le Corbusier l'affirme clairement dans une interview des années Soixante “...je me suis aperçu qu'en confiant mes émotions à un objectif j'oubliais à les faire passer par moi, ce qui était grave, alors j'ai laissé

\footnotetext{
${ }^{7}$ Fondation Le Corbusier: Voyages de jeunesse: L5-1-124.
} 
tomber la Kodak et j'ai pris mon crayon et depuis, j'ai toujours dessiné tout et n'importe où"8. Quoi qu'il soit l'outil d'enregistrement, Le Corbusier semble déjà appréhender, lors de son voyage dans les Balkans, les avantages d'un point de vue suffisamment éloigné et détaché, à même de mieux faire comprendre les relations entre l'architecture et le milieu naturel/urbain dans lequel elle s'insère. Ces différentes images ne contiennent pas encore de propos liés au projet de transformation du site, l'objectif étant, pour l'instant, celui de la documentation, voire de la compréhension sans visée opérationnelle. Les croquis et les dessins du jeune architecte fixent, par des traits rapides, les lignes principales du paysage dans toute son étendue. Dans l'un des nombreux panoramas d'Istanbul, par exemple, Le Corbusier souligne par des rayures horizontales la mer et quelques collines, alors que les lignes verticales sont essentiellement réservées aux bâtisses, telles que mosquées ou bâtiments remarquables par leur profil $1^{9}$. La présence, au premier plan, de l'eau, semble prendre un sens bien précis. En effet, les vues représentées depuis le bateau en mer - à moins qu'il s'agisse, parfois, de dessins élaborés depuis la côte d'en face - accordent une place énorme à cette surface plane et immobile, accentuant parlà, non seulement la distance physique de la terre, mais aussi le détachement de l'observateur et sa capacité à saisir l'essentiel de façon synthétique, comme en témoigne un second panorama d'Istanbul ${ }^{10}$.

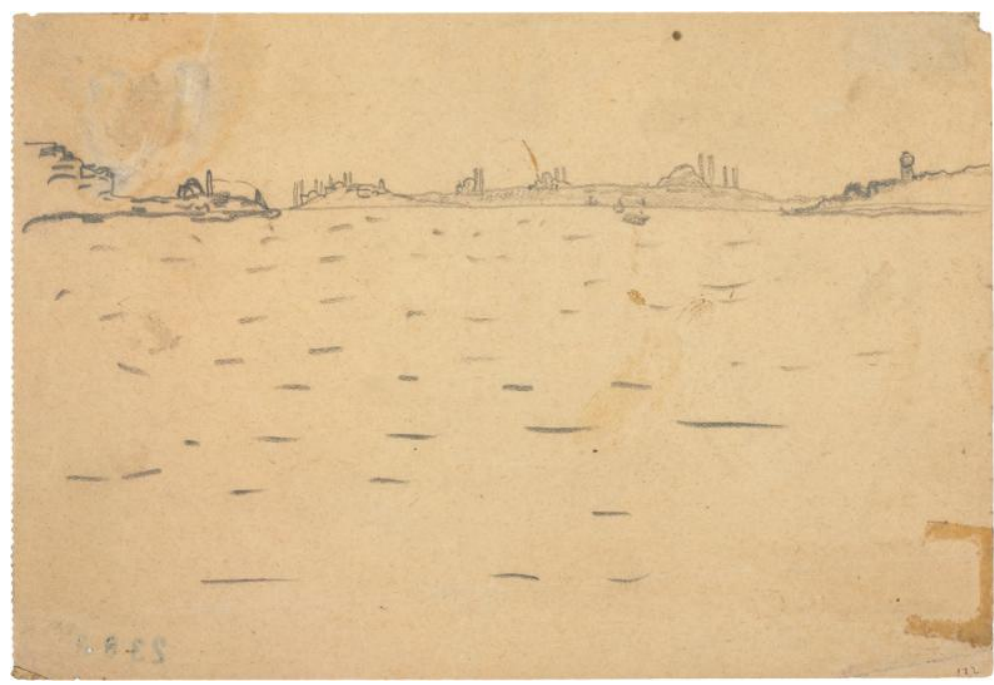

3. Panorama d'Istanbul, 1911. FLC, 2383.

Dans celui-ci le cadrage repousse très loin la ligne de l'horizon avec le skyline bidimensionnel de la ville sur le Bosphore. Le Corbusier utilise la plus grande partie de carnet pour y dessiner la mer par quelques touches de crayon. Une surface qui n'est donc presque jamais exploitée pour ses reflets séduisants, mais qui ne sert qu'à faire reculer le profil urbain au fond du dessin. Le ciel et la mer occupent ainsi la plus grande partie des croquis élaborés par le Corbusier depuis un bateau. Le skyline de la ville qui surplombe l'eau devient juste une trace horizontale au milieu d'un environnement naturel qui l'entoure totalement. En ce qui concerne Rio, Le Corbusier écrit en 1930 "La ville s'annonçait par une ligne qui, seule, est capable de chanter avec le caprice véhément des monts: l'horizontale"11.

Son approche et son appréhension des agglomérations surplombant l'eau, deviennent de plus en plus liées et conditionnées par le moyen de transport maritime ou fluvial qui façonne le premier aperçu visuel. L'élaboration des énormes infrastructures surélevées envisagées pour Rio ou Alger, trouve en effet son origine dans cette ligne

\footnotetext{
${ }^{8}$ Cité par Benton, Tim: "Le Corbusier photographe secret". In Herschdorfer, Nathalie et Umstätter, Lada (Dir.): Construire l'image. Le Corbusier et la photographie. Paris: Textuel, 2012, pp. 30-53, cit. p. 49.

${ }^{9}$ Panorama d'Istanbul, 1911, FLC 2459.

${ }^{10}$ Panorama d'Istanbul, 1911, FLC 2383.

${ }^{11}$ Le Corbusier: Précisions...op. cit., p. 245.
} 
d'horizon toujours accentuée dans ses premiers relevés. Bien qu'il s'agisse d'interventions de grande envergure et d'une emprise à échelle territoriale, la démarche qui les sous-tend paraît être celle qui veut le moins modifier et altérer le sol ainsi que le visage et la silhouette générale du site saisis depuis la mer, en s'étendant essentiellement sur l'horizontalité. Les skylines sont alors dessinés par Le Corbusier, non seulement pour fixer le premier aperçu d'une ville, mais aussi pour vérifier la correcte insertion du projet. La démarche utilisée à Rio de Janeiro, avait déjà été expérimentée lors d'un projet, en 1927, pour un autre site surplombant l'eau. C'est ainsi que la vue du Palais des Nations élaborée depuis le lac de Genève a l'avantage d'être le fruit d'un relevé pris avec précision depuis une place suffisamment reculée pour pouvoir souligner, comme l'explique la légende, que "la géométrie des formes architecturales entre en symphonie avec les richesses naturelles du site" temps, la représentation du projet vu du lac vise à accentuer, par le biais de l'eau qui sépare l'ensemble architectural du bateau (imaginaire ou réel), la distance spatiale et mentale entre l'observateur et l'objet en question. Cette recherche de distance objectivante s'aligne parfaitement avec la représentation pleine-page à travers la vue cavalière plongeante que Le Corbusier utilise pour illustrer ce projet: une axonométrie à même de renforcer l'effet d'abstraction et de séparation. Cette mise à distance recherchée par l'architecte depuis ses premiers voyages en bateau, trouvera son aboutissement naturel, comme on aura l'occasion de voir, dans les survols des villes en avion. La séquence sténographique reprise depuis le bateau près de Manhattan et publiée en 1937 en guise de conclusion dans Quand les cathédrales étaient blanches veut aussi souligner le "paysage émouvant" de la ville américaine ${ }^{13}$. La réalisation des huit croquis dans lesquels se croisent les éléments de l'embarcation avec la silhouette des gratte-ciels, a été rendue possible par le rythme lent du moyen de transport.

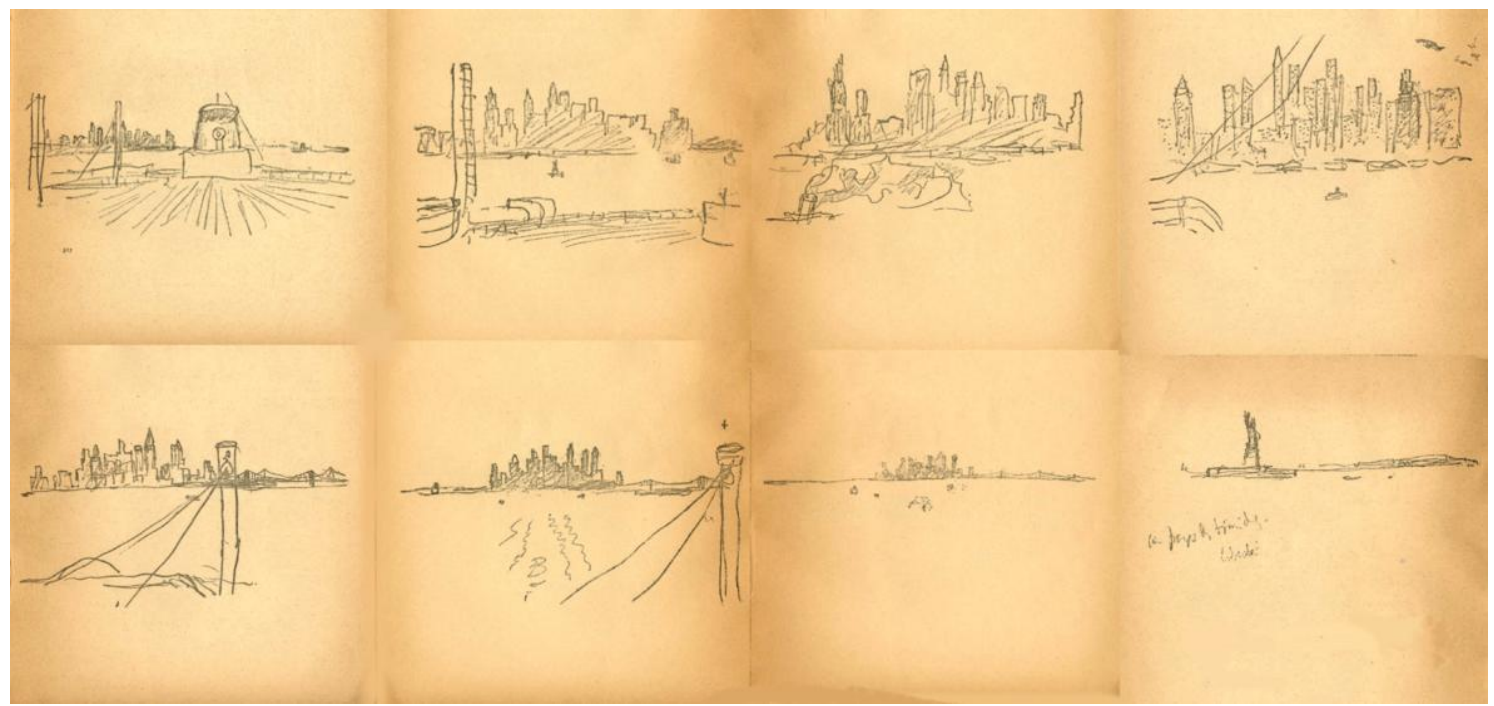

4. Séquence sténographique de Manhattan. Le Corbusier: Quand les cathédrales étaient blanches. Voyage au pays des timides. Paris: Plon, 1937, pp. 315-322.

Le procédé qui semble viser la "vue panoramique animée" sera repris en 1936, par un outil iconographique différent, lorsque Le Corbusier, de retour du Brésil, réalisera des photos prises en séquence depuis le paquebot Conte Biancamano ${ }^{14}$. Le lien avec le medium cinématographique devient de plus en plus évident.

La vue depuis les bateaux, ne permet pas seulement à Le Corbusier d'élaborer une première impression fixée sur le papier ou la pellicule photo à travers les lignes quelque peu abstraites du skyline de la ville qui

\footnotetext{
${ }^{12}$ Le Corbusier: Vers une architecture. Paris: Arthaud, 1977, p. XIV. Cf. le chapitre «Température », ajouté en 1928 à l'occasion de la troisième édition.

${ }^{13}$ Le Corbusier: Quand les cathédrales étaient blanches. Voyage au pays des timides. Paris: Plon, 1937, pp. 315-322.

${ }^{14}$ Cf. Benton, Tim: LC Foto: Le Corbusier Secret Photographer. Zürich: Lars Müller, 2013.
} 
appréhendent les relations entre architecture historique, projet, espace urbain, paysage, territoire. Elle autorise aussi, une fois que le navire s'approche de la côte, une vision plus rapprochée où les principales émergences architecturales sont enregistrées afin de noter les points fragiles et délicats où il faudra intervenir. Il s'agit toujours de visions frontales, mais capables, par leur proximité au front de mer, de faire jaillir des considérations ponctuelles sur l'aménagement urbain actuel et futur. Cette approche déjà riche en propositions, devra toutefois être validée et confrontée par les "pieds du marcheur" avec la découverte des espaces plus cachés et retirés de la ville, ainsi que par le survol aérien que Le Corbusier découvre à la fin des années Vingt. C'est en suivant ces deux étapes que se déroule, par exemple, son approche de la côte newyorkaise à bord du Normandie. Sa première impression depuis le paquebot attendant la "quarantaine" est celle d'une ville fantastique, "quasi mystique": le "temple du nouveau monde"." Puis, lorsque l'embarcation atteint une distance réduite du front de mer, "l'apparition se transforme en image d'une brutalité et d'une barbarie inouïe" ${ }^{\prime 16}$. Le premier skyline permet d'élaborer un aperçu visuel principalement lié aux sensations et aux émotions; la vision frontale rapprochée autorise les jugements et les commentaires critiques que la promenade en ville accomplie le soir et les photos aériennes qui accompagnent la publication de Quand les cathédrales étaient blanches pourront confirmer ou contredire.

Il s'agit donc d'un regard qui sous-tend déjà une approche analytique et critique du site, traduit par des croquis où nombreuses sont aussi les annotations écrites, souvent à mi-chemin entre constat et projet. Les vues de Barcelone depuis la mer tracées en 1932 constituent peut-être la démonstration la plus évidente que ces représentations accompagnées de commentaires - ne font plus vraiment partie de la culture du voyage, comme l'a justement souligné Giuliano Gresleri ${ }^{17}$. Le Corbusier note sur l'un de ses croquis: "Il faudrait gratte-ciel même hauteur que Monjuich"18.

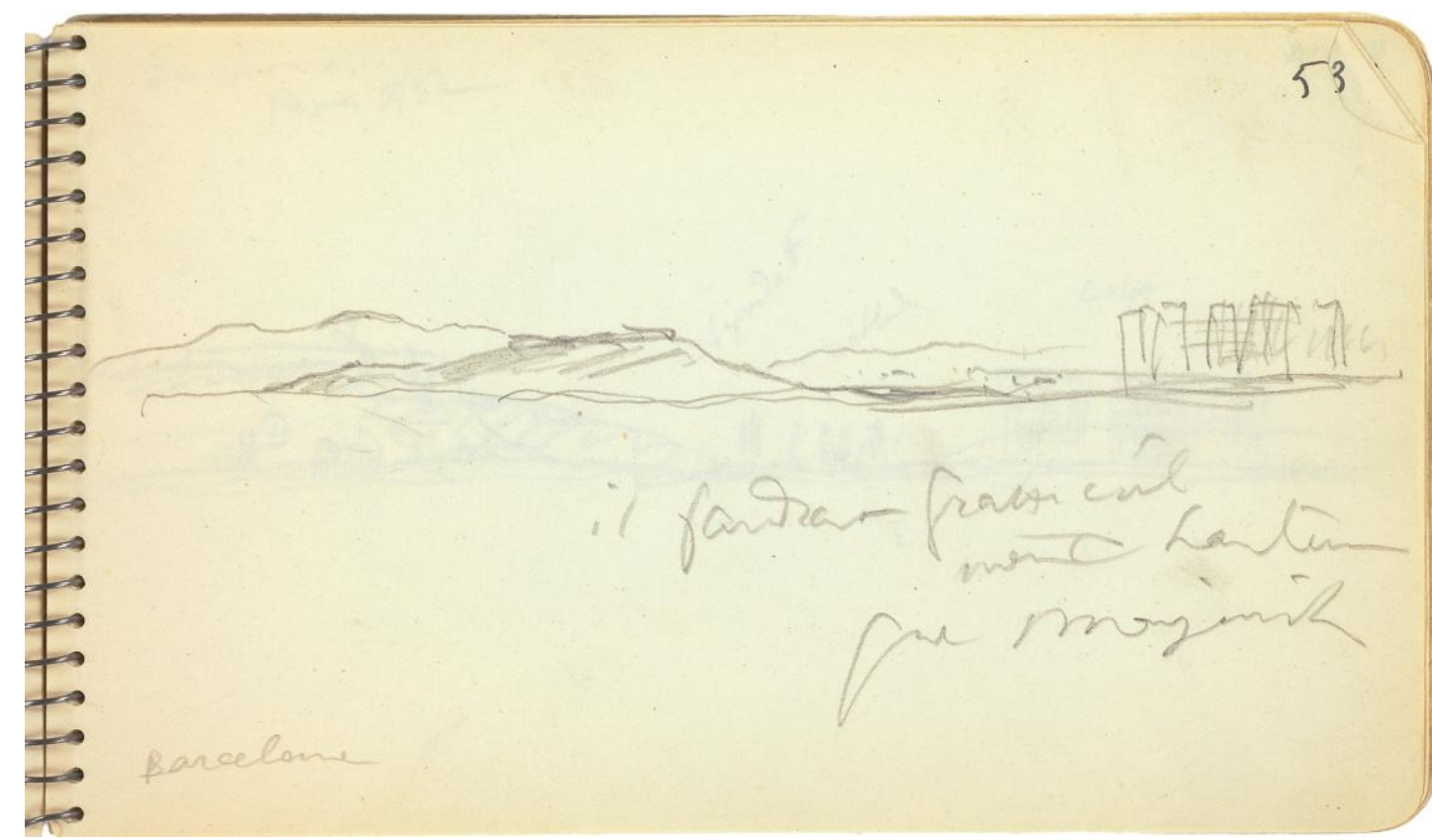

5. Vue de Barcelone depuis la mer, 1932. FLC, carnet C10, n. 636.

\footnotetext{
${ }^{15}$ Le Corbusier: Quando le cattedrali erano bianche. Faenza: Faenza editrice, 1975, p. 42.

${ }^{16}$ Ibid., p. 42

${ }^{17}$ Cf. l'interprétation par Gresleri: Gresleri, Giuliano: "Dal diario al progetto. I Carnet 1-6 di Le Corbusier". In Lotus, N. 68. 1991, pp. 6-21.

${ }^{18}$ FLC, carnet C10, n. 636.
} 
La même visée opérative est très explicite dans le dessin où l'architecte esquisse le skyline de la ville catalane avec ses différentes hauteurs ${ }^{19}$, ou dans celui qui représente Rio de Janeiro en $1936^{20}$ où se lisent les différentes émergences architecturales et naturelles, ainsi que leur impact sur le paysage global du front de mer. Dans le compte rendu de son séjour brésilien, Le Corbusier déclare apprécier la perspective obtenue depuis le bateau au milieu de la baie: "Or, du large de Rio, j'ai repris mon carnet de dessin; j'ai dessiné les monts et, entre les monts, l'autostrade future et la grande ceinture architecturale qui la porte... Les paquebots qui passaient, immeubles magnifiques et mouvants des temps modernes, trouvaient là-bas, suspendus dans l'espace au-dessus de la ville, une réponse, un écho, une réplique"21.

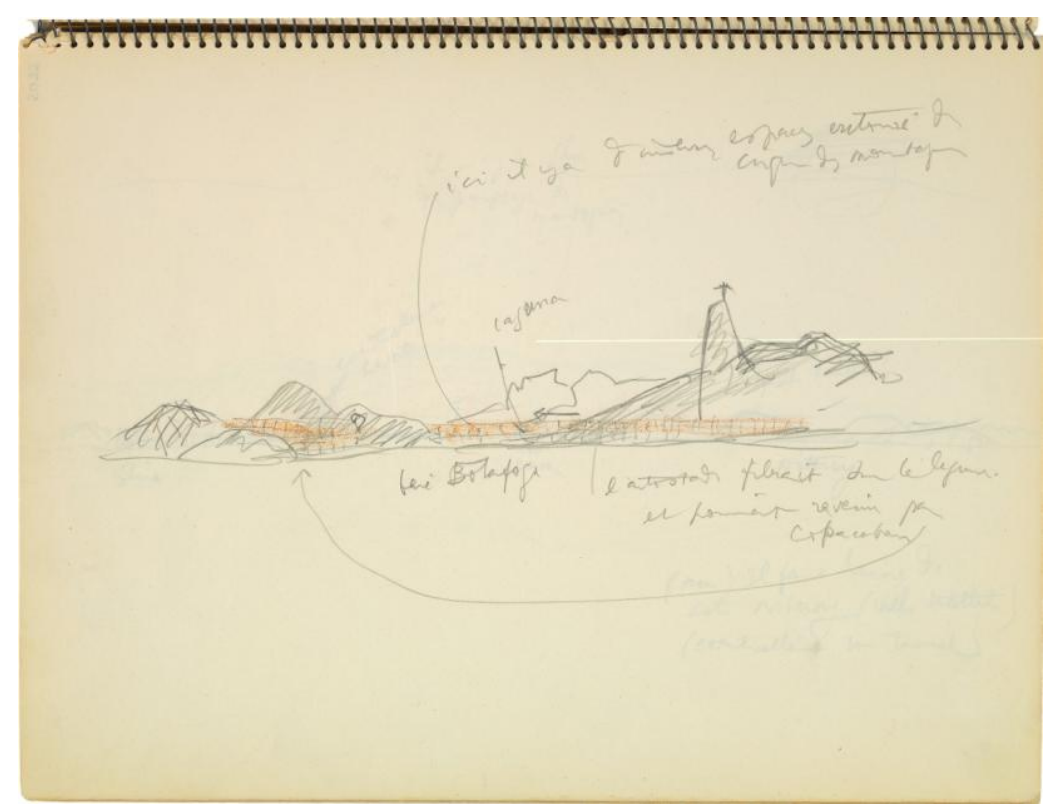

6. Un des premiers croquis pour Rio de Janeiro. FLC, dessin 5033.

Cette sorte de résonnance acoustique qui se crée entre le navire et l'architecture semble confirmer à Le Corbusier que son projet remplit toutes les conditions pour aboutir. Elle lui permet aussi de réfléchir sur la complémentarité de l'approche maritime et du survol aérien qui se disputent gentiment le rôle principal dans l'appréhension et l'interprétation des lieux urbains grâce à un enregistrement des données qui se produit de façon systématique et calme: "Du large, j'ai vu dans mon esprit la ceinture ample et magnifique des bâtisses, avec le couronnement horizontal de l'autostrade frappant de mont en mont et tendant la main d'une baie à l'autre. L'avion est prêt à en devenir jaloux; de telles libertés lui semblaient réservées" ${ }^{\prime 2}$. Mais si la vue horizontale de la ville maritime autorisée par le bateau est à même d'enregistrer l'insertion dans le site et l'unité paysagère, la perception du haut d'un avion est manifestement celle qui permet de comprendre l'échelle territoriale, en devenant, pour Le Corbusier, la seule capable de dévoiler les véritables secrets et les grandes leçons que l'esprit humain pouvait élaborer seulement de façon intuitive ${ }^{23}$.

\footnotetext{
${ }^{19}$ FLC, carnet C10, n. 637

${ }^{20}$ FLC, carnet C12, n. 731.

${ }^{21}$ Le Corbusier: Précisions... op. cit., p. 244.

${ }^{22}$ Ibid., p. 244.

${ }^{23}$ Dans une note prise en 1955 en survolant l'Inde, Le Corbusier écrit : "De même que des perspectives de rivières-méandres étaient du Léonard (sic! Piero della Francesca). Il y a eu des scénarios de nuages que certains ont peint, les ayant imaginés vus du haut des airs" (FLC, carnet J39, n. 448).
} 


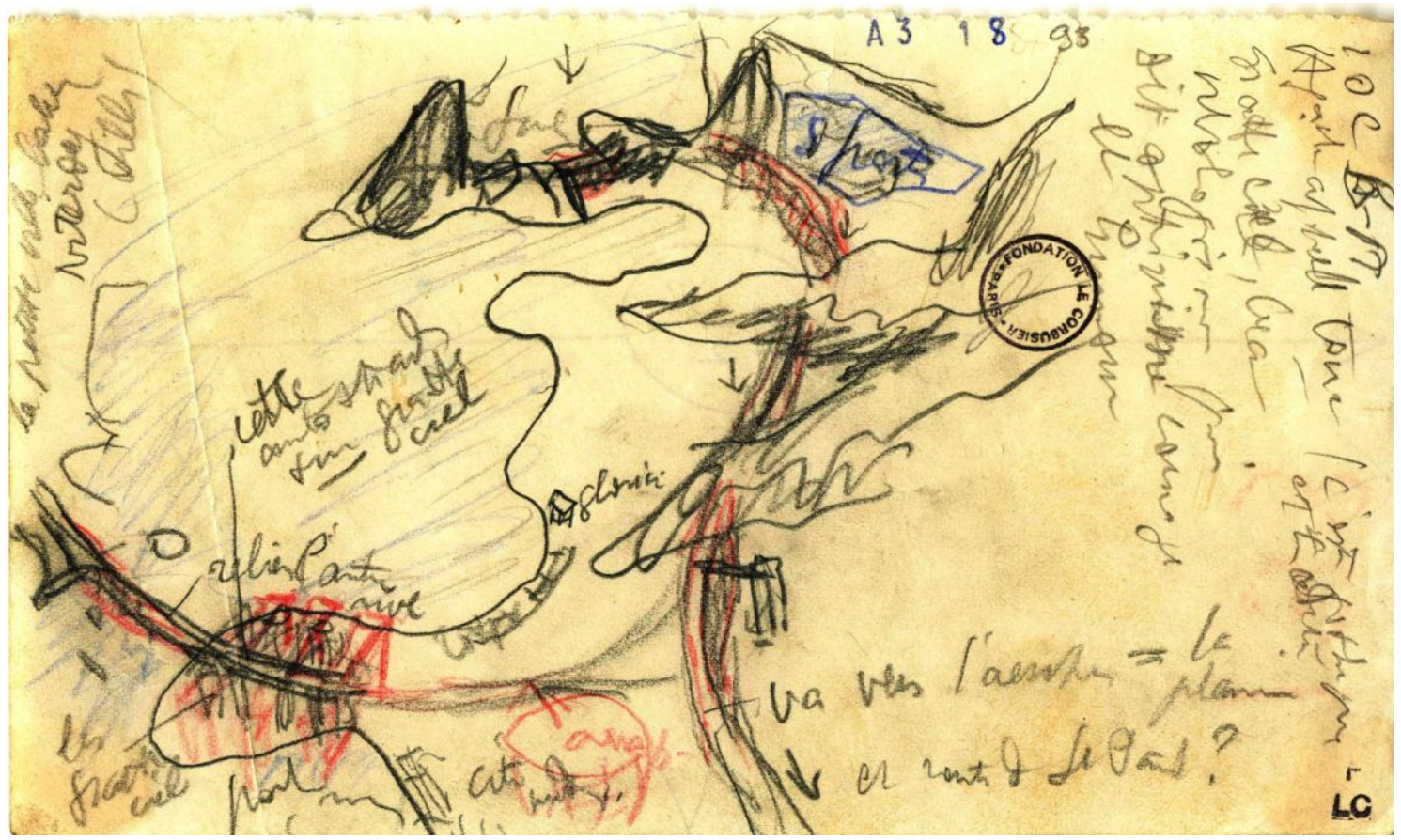

7. Rio de Janeiro, vue depuis l'avion avec croquis de son projet pour la ville. FLC, A3.18.98.

\section{Comment découvrir les secrets d'une ville}

À partir de ses premiers voyages, Le Corbusier commence à apprécier la vue privilégiée déjà par Montaigne lors de sa découverte des villes. À Naples, par exemple, après avoir visité l'église du Jésus, il se dirige vers les hauteurs, en direction de la route de Posillipo, depuis laquelle il peut contempler - comme le suggérait le célèbre guide d'Italie de Baedeker utilisé par beaucoup de voyageurs de l'époque - "les plus magnifiques points de vues, tout particulièrement beaux au coucher du soleil; aucun étranger ne devrait négliger d'y faire une promenade"24. Depuis cette colline Le Corbusier est désormais à même d'admirer toute la partie orientale du golfe et de signaler les quelques opérations ponctuelles - comme le site industriel de l'Ilva - venues altérer l'horizontalité du paysage $\mathrm{e}^{25}$. À Rio de Janeiro, en 1936, il va jusqu'à réaliser une séquence de deux minutes de film depuis la plateforme sommitale du Morro da Providencia, en enregistrant le paysage de la grande baie ${ }^{26}$. Ses achats de cartes postales avec des vues plongeantes ou aériennes répondent aussi, comme on le verra, à ce besoin d'embrasser d'un coup d'œil le panorama construit et naturel. Dans ses publications, Le Corbusier commence également à faire appel aux photographies prises du haut ou depuis les appareils qui s'envolent au-dessus des villes. Dans le n. 18 de l'Esprit nouveau, par exemple, il publie deux clichés, dont l'un représente une vue aérienne de New York ${ }^{27}$. Pour le frontispice de L'art décoratif d'aujourd'hui (1925) Le Corbusier a recours à la photo zénithale publiée par la revue Stavba ${ }^{28}$. La même année, il utilise pour un chapitre d'Urbanisme une carte postale dont le titre est déjà révélateur: Paris au début $d u$ XXe siècle. Une promenade en dirigeable ${ }^{29}$. Le Corbusier est déjà pleinement conscient que l'aviation ne constitue pas seulement un progrès technique, mais qu'elle sera à l'origine d'une

\footnotetext{
${ }^{24}$ Baedaker, Karl: L'Italie des Alpes à Naples. Paris: Ollendorgg, 1909, p. 393.

${ }^{25}$ Cf. Fatigato, Orfina: "À travers Naples". In A.A.V.V.: L'invention d'un architecte. Le voyage en Orient de Le Corbusier. Paris: Éditions de la Villette, 2013, pp. 426-439.

${ }^{26}$ Cf. Prelorenzo, Claude: “Gammes cinématographiques". In Le Corbusier. Aventures photographiques. Paris, Éditions de la Villette, 2014, pp. 70-87.

${ }^{27}$ Cf. l'article "L'ordre" écrit par Le Corbusier dans le numéro 18 de l'Esprit nouveau. À ce sujet cf. Mazza, Barbara: Le Corbusier... op. cit., p. 98.

${ }^{28}$ Mazza, Barbara: Le Corbusier... op. cit., p. 99

${ }^{29}$ Ibid, p. 101.
} 
nouvelle manière de voir le monde. Ce n'est pas un hasard s'il envisage la publication - malheureusement jamais réalisée - d'un paragraphe/chapitre entier consacré aux vues depuis l'avion. La Fondation Le Corbusier conserve en effet un précieux document où l'on lit "faire 1 paragraphe sur la vue d'avion nouvel état de chose dans l'esthétique urbaine comme sensation architecturale. Montrer alors quelques vues d'avions (service topographique) de Paris $^{\prime \prime 30}$. Les trois croquis qui accompagnent ce propos, représentent des vues plongeantes - aucune vue zénithale du même genre que celle utilisée dans son frontispice de 1925 n'y figure - semblables à celles qu'un avion en phase d'atterrissage ou de décollage pourrait enregistrer. Des images qui, tout en saisissant l'étendue des différentes villes, visent à faire ressortir les volumes et les silhouettes des bâtiments principaux de la trame urbaine, de façon très réaliste et peu géométrisée. Dans un dessin l'on croit apercevoir les toits de Notre-Dame et du Panthéon, alors qu'un autre, légendé Chicago, affiche un seul gratte-ciel au milieu de lignes à peine ébauchées.

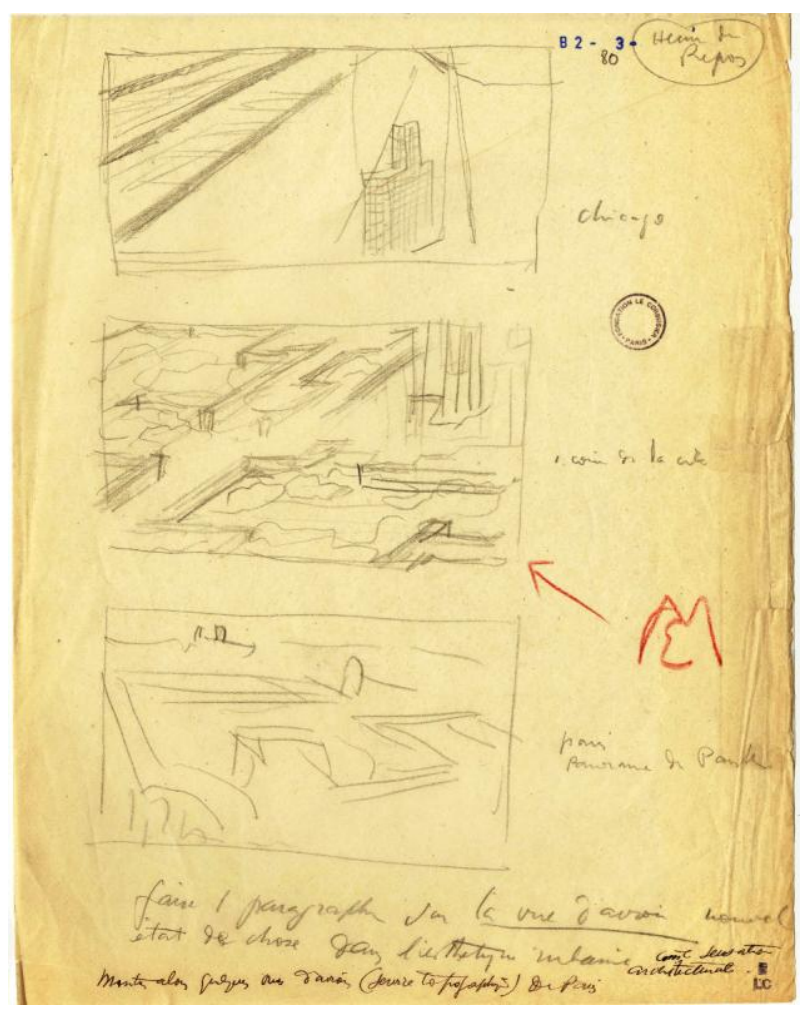

8. Feuille esquissant le projet de rédiger un chapitre d'Urbanisme sur la vue d'avion

Le Corbusier n'a pas encore découvert par ses propres yeux le vol en avion et ses dessins ressemblent davantage aux vues perçues depuis le sommet d'une colline (ou d'un édifice) qu'aux visions territoriales et planétaires dont il fera l'expérience quelques années plus tard. En effet, son rêve de voler se concrétise une première fois en 1928 lors d'un voyage en avion en direction de Moscou, mais trouve son véritable exaucement au cours des années 1929-1933 grâce aux nombreux survols des villes et du territoire de l'Amérique du sud et de l'Algérie. Les enregistrements aussi bien sur ses carnets de croquis que sur la pellicule photo ne se feront pas attendre. Des images qui, comme les notes qui très souvent les accompagnent, atteignent une plus grande capacité de synthèse et de concision. Entre ses phrases lapidaires, ses esquisses à peine ébauchées, ses photos instantanées et la vitesse du nouveau moyen de transport, existe sans doute un lien très fort ${ }^{31}$. La réalité est enregistrée sans médiations, dans son authenticité et avec promptitude. Ce qui ne signifie absolument pas que la saisie perde en précision. Tout au contraire, la vision d'avion est, selon l'architecte "la plus calme, la plus régulière, la plus

\footnotetext{
${ }^{30}$ FLC, B2-3-80.

${ }^{31}$ Cf. Bruno Pedretti: "Il volo dell'etica”. In Casabella. № 531-532. Janvier-Février 1987, pp. 74-85.
} 
précise qu'on puisse désirer: on apprécie le pelage tacheté ou rouge ou noir d'une vache. Tout prend la précision d'épure, le spectacle n'est pas hâtif mais très lent, sans rupture... "32. Car au fond la vitesse de l'aéroplane, sa "course foudroyante... donne la sensation étrange de la presque immobilitế”33.

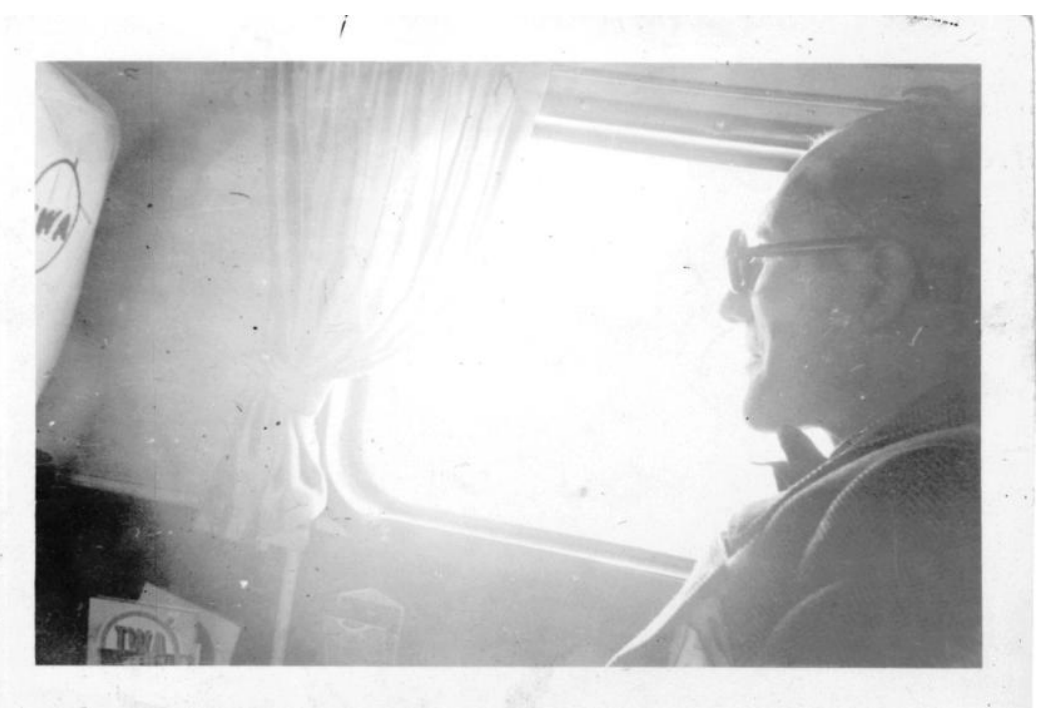

9. Le Corbusier lors d'un voyage en avion. FLC, L5-2.41.

La compréhension profonde des villes et des territoires de la part de Le Corbusier se fera par les deux modalités de perspective aérienne, complémentaires: celle "des paquebots de l'air qui foncent à 6000 mètres d'altitude par-dessus océans, savanes, sierras ou cordillères" et qui de très haut saisit la morphologie et les grands signes du territoire, mais aussi celle qui est autorisée par l'avion dans ses phases de décollage ou d'atterrissage ou bien par les petits appareils ou encore par ce qu'il nommera l'“aéronautique verticale", à savoir les hélicoptères qui, "jouant à l'hippocampe des aquariums, montent et descendent de-ci de-là des montagnes sans aéroport, sur des aires d'atterrissage minuscule" ${ }^{\text {34 }}$. A titre d'exemple, il suffit de rappeler la demande de l'architecte adressée au pilote lui montrant Sao Paulo depuis l'avion, à savoir de voler aussi bas que possible. Dans ce cas précis le but était d'arriver à apprécier les hauteurs et la spatialité du tissu urbain, avec ses volumes et ses imbrications, l'important étant toujours d'appréhender au mieux le site survolé. Une expérience semblable est relatée par le Corbusier en 1935: le pilote qui l'avait accompagné en mars 1933 au-dessus du Mzab, ne se serait pas limité à survoler le territoire algérien, mais serait descendu sur les villes pour montrer à l'architecte "the principle of the towns" ${ }^{\prime 3}$. C'est alors, du haut de l'avion, que le Corbusier découvre le secret des villes du désert avec les jardins et les arcades dont se composent les maisons locales ${ }^{36}$.

\footnotetext{
${ }^{32}$ Le Corbusier: Précisions... op. cit., p. 8.

${ }^{33}$ Le Corbusier: Sur les 4 routes. Paris: Denoël, 1970 (1 ${ }^{\text {ère }}$ éd. 1941), p. 42.

${ }^{34}$ Le Corbusier: "Urbanisme et Aéronautique". In Techniques et Architecture. № 9-12, 1947, reproduit in: Duboy, Philippe: Le Corbusier. Croquis de voyages et études. Paris: La Quinzaine Littéraire-Louis Vuitton, 2009, p. 320-323, cit. p. 322.

${ }^{35}$ Le Corbusier: Aircraft... op. cit., p. 12.

${ }^{36}$ Ibid. p. 12.
} 


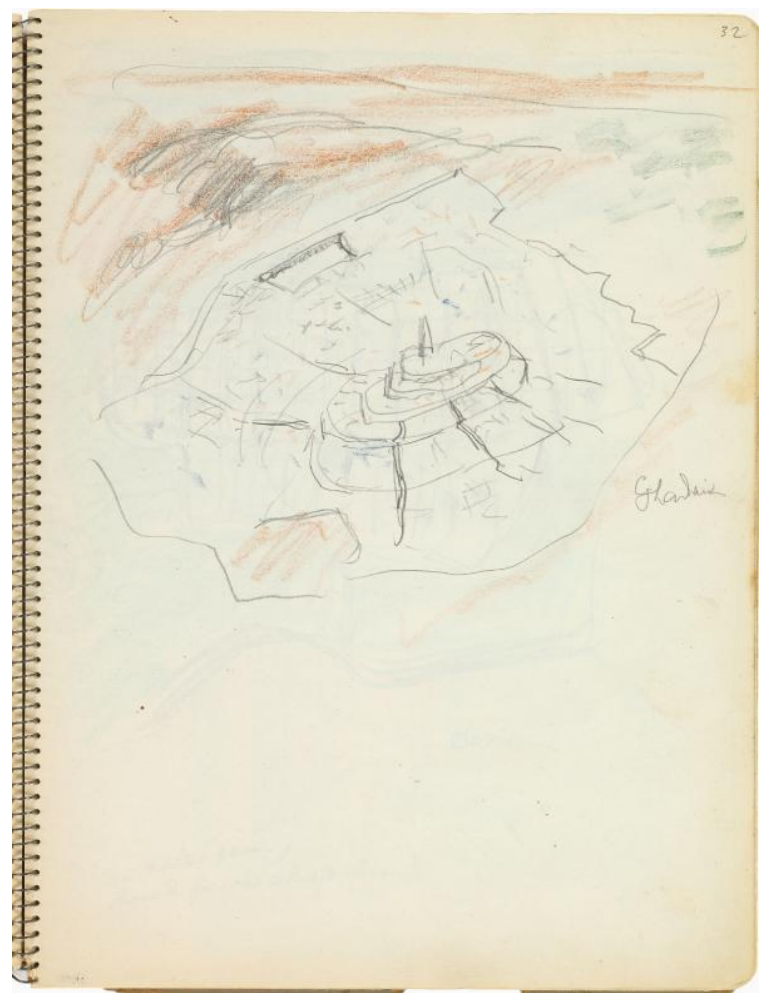

10. Vue aérienne d'une ville du désert, 1933. FLC, dessin 5010.

Par ailleurs, Le Corbusier revendique la nécessité de "voir" et de ne pas se limiter à l'acte, qu'il juge passif, du simple regard. Il semble partager avec l'un de ses plus célèbres prédécesseurs, Viollet-le-Duc, la conviction que "voir c'est savoir" ${ }^{\text {"37 }}$. Au bout de ses nombreux voyages intercontinentaux, l'architecte francosuisse aboutit à la conclusion que "l'avion ... voit tout minutieusement" seul moyen capable de découvrir les secrets des villes. Le meilleur outil d'enregistrement de ces visions "actives" et "conscientes" demeure, selon lui, le dessin: un geste manuel mais aussi un acte intellectuel de connaissance et de sélection, qui n'est pas sans rappeler, encore une fois, les réflexions du célèbre théoricien français du XIXe siècle pour qui dessiner trouvait une équivalence dans l'étude et non dans la simple reproduction. "Je n'existe dans la vie qu'à condition de voir", écrit le Corbusier de retour du Brésil en $1929^{39}$. C'est d'ailleurs peut-être cette conviction que la vue aérienne puisse conduire à appréhender le sens le plus profond des choses, qui intensifie le plaisir du voyage en avion chez Le Corbusier au point de l'entendre affirmer "me voici au Paradis dans l'air comme un oiseau" 40 ou encore "je ne suis jamais si tranquille que dans l'avion. Cosmos et solitude amicale et bénéfique" ". Ses lectures, pendant ses traversées européennes aussi bien que transatlantiques, traduisent parfaitement cet état d'esprit voué à la méditation, aux réflexions sur le sens de la vie et de l'univers entier. Rabelais, définis comme "homme qui apporte le courage et la pensée ${ }^{\text {,42 }}$, Machiavel et $\mathrm{Brecht}^{43}$, ne constituent que quelques exemples des auteurs qui accompagnent l'architecte à 5000-6000 mètres de hauteur. Don Quichotte se retrouve souvent entre ses mains, l'incitant peut-être à lire la réalité avec d'autres yeux. Et le regard de Le Corbusier se fait de plus en plus attentif,

\footnotetext{
${ }^{37}$ Viollet-le-Duc, Eugène-Emmanuel: Histoire d'un dessinateur. Comment on apprend à dessiner. Paris: Hetzel, 1879, p. 302.

${ }^{38}$ Le Corbusier se trouve sur l'avion Bombay-Dehli en 1955: FLC, carnet J39, 439.

${ }^{39}$ Le Corbusier: Précisions... op. cit., p. 8.

${ }^{40}$ Le Corbusier: Carnets 2. 1950-1954. Paris: Herscher, 1981, n. 934.

${ }^{41}$ FLC, carnet E20, n. 422

${ }^{42}$ FLC, carnet J36, n. 268.

${ }^{43}$ FLC, carnet L47, n. 881 .
} 
sélectif, analytique, précis, étendu, au point de balayer l'espace cosmique et d'en tirer des conclusions graphiques ou écrites qui peuvent parfois paraître laconiques et lapidaires à cause de leur synthèse poussée à l'extrême.

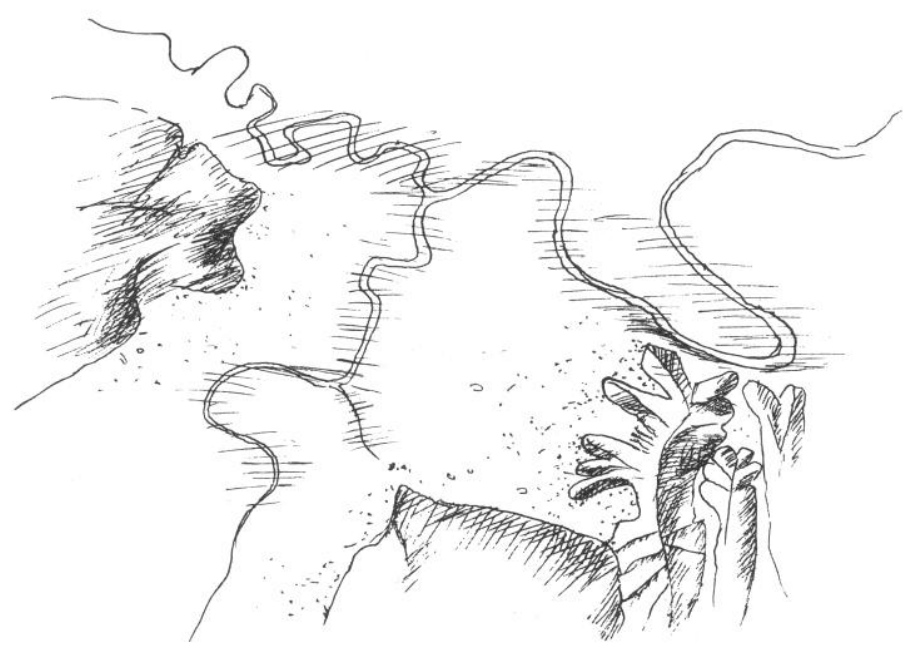

11. Paysage algérien depuis l'avion, 1933. Le Corbusier: Aircraft. London: The Studio, 1935, p. 116.

\section{Analyse et projet: la naissance d'une nouvelle échelle}

La précision autorisée par la vue aérienne devient, chez Le Corbusier, le point de départ d'une série de projets de transformation d'une réalité qui lui apparait, du haut de l'avion, particulièrement riche en contradictions. Le spectacle offert par l'aéroplane - "Quel sinistre bilan!" et de l'aménagement urbain. En 1930, dans Précisions l'architecte avait souligné la capacité de l'avion d'entrer "dans l'intimité de la ville" et de lui arracher "d'un simple coup d'œil d'oiseau planeur tous les secrets qu'elle cachait si facilement au pauvre terrien sur ses deux pieds" ${ }^{\prime 4}$. Mais la compréhension se transforme très rapidement en jugement critique. Les considérations que l'architecte formule en 1935 dans le texte qu'il écrit pour accompagner les photos que la célèbre maison d'édition anglaise The Studio envisageait de publier dans un volume consacré à la vision aérienne, sont très éloquentes à ce propos ${ }^{46}$. "The airplane is an indictment. It indicts the city. It indicts those who control the city. By means of the airplane, we now have proof, recorder on the photographic plate, of the rightness of our desire to alter methods of architecture and town-planning, ${ }^{, 47}$. Ce nouvel état de lucidité incite "to change something in the present world" les vieilles villes et de les reconstruire. La vision claire et lucide constitue l'acte préalable à l'intervention: "The airplane has given us the bird's-eye view. When the eye sees clearly, the mind makes a clear decision" "Thès nombreuses sont les annotations qui accompagnent les croquis de Le Corbusier effectués lors de ses voyages en avion dans lesquelles il exprime ses pensées, voire ses condamnations des solutions urbaines adoptées dans le monde entier. La France figure comme l'un des pays le plus incriminé où le "parcellement actuel des terres" est

\footnotetext{
${ }^{44}$ Le Corbusier: Sur les $4 \ldots$ op. cit., p. 150.

${ }^{45}$ Le Corbusier: Précisions... op. cit., p. 235.

${ }^{46}$ Le Corbusier: Aircraft... op. cit.

${ }^{47}$ Ibid, p. 11.

${ }^{48}$ Ibid, p.11.

${ }^{49}$ Ibid, p. 13.
} 
“affolant", "désastreux" "50. En 1962, en survolant la capitale il note "la banlieue de Paris vue d'avion est un scandale, une maladiel/ toutes maisons se touchent mais se voient, s'entendent..." ${ }^{.1}$. La vieille Europe, en générale, n'échappe pas à ses critiques féroces: des expériences allemandes des cités jardins (à Frankfort par exemple) ${ }^{52}$ à "l'extrême cacophonie" de Barcelone ${ }^{53}$. Malgré l'emploi de matériaux de construction peu modernes, Le Corbusier semble préférer les solutions adoptées dans les pays du Moyen Orient qu'il découvre en avion comme par exemple la ville d'Abadan, en Iran: située près d'un "fleuve majestueux", elle est une "ville moderne en torchis mais d'une géométrie et ordonnance totale (du logis)" où même les "tanks aluminiumés groupés par quatre... [sont] dans un ordre magnifique" ${ }^{\text {54 }}$. Font aussi partie de cette appréhension des villes et du territoire survolés, les remarques et les observations sur la couleur de la terre, des sites, de l'horizon. Dans ses dessins, mais surtout dans les notes qui les accompagnent, Le Corbusier transcrit et commente les gradations et les couleurs lui permettant de mieux comprendre l'humidité du sol et par là "toute une biologie", toute cette "vie organique" qui se dévoile à ses yeux à 5000-6000 mètres de hauteur". L"“herbe brulée de l'horizon", ", les "sables violacés et roses, les eaux verts pâles" de l'estuaire de l'Indus ${ }^{57}$, contribuent à saisir la spécificité du territoire examiné depuis le ciel. Une fois que l'architecte pense avoir réussi à entrer "dans le corps et le cœur de la ville" et avoir "compris une part de son destin..." "58, jaillit alors la "joie de la création",59. Un sentiment qui vient remplacer la "joie des sens",60 provoquée par la vision du monde à ses pieds.

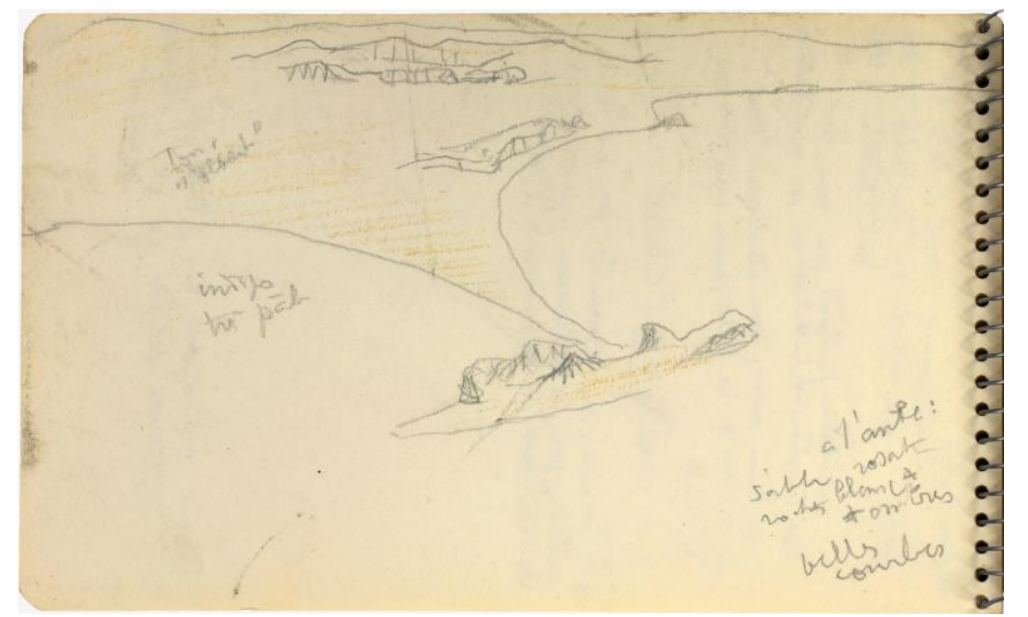

12. Depuis l'avion, Le Corbusier note les couleurs des territoires survolés, 1958. FLC, carnet J37, n. 334.

Du retour d'Amérique du sud en 1929, à bord du paquebot qui le ramène en Europe et où il est en train d'écrire Précisions, Le Corbusier aboutit à des conclusions claires et rigoureuses concernant son devoir d'architecte du XXe siècle. Depuis qu'il a découvert un pays "dimensionné pour l'avion"61 pour lequel il a élaboré des projets de grande envergure à échelle territoriale - à Buenos Aires comme à Rio de Janeiro - il estime que son attention et ses préoccupations devront désormais s'adresser essentiellement aux questions urbaines et aux territoires

\footnotetext{
${ }^{50}$ FLC, carnet 70, n. 1062.

${ }^{51}$ FLC, carnet S66, n. 866.

${ }^{52}$ FLC carnet S67, n. 934.

${ }^{53}$ FLC, carnet C10, n. 645.

${ }^{54}$ FLC, carnet K45 n. 802.

${ }^{55}$ Le Corbusier: Précisions... op. cit., p.7.

${ }^{56}$ FLC, carnet J37, n. 365.

${ }^{57}$ FLC, carnet J37, n. 366.

${ }_{58}^{58}$ Le Corbusier: Précisions... op. cit., pp. 235-236.

${ }^{59}$ Ibid, p. 236.

${ }^{60}$ FLC, cahier de dessins n. 5, année 1933, p. 19. Cf. aussi Aircraft... op. cit.: "No longer a delight of the senses", p. 123.

${ }^{61}$ Le Corbusier: Précisions... op. cit., p. 3.
} 
métropolitains. Le Corbusier est de plus en plus conscient que grâce à ce nouveau moyen de transport "on est devenu un animal nouveau sur la planète ${ }^{, 62}$ et que ce genre de vision depuis les hauteurs ne peut plus utiliser les catégories analytiques traditionnelles mais demande une nouvelle approche du regard. Dans une note prise en 1962 sur le vol Nice-Paris, il réfléchit à la naissance d'une nouvelle d'appréhension du monde, qui n'est plus proportionnée à la hauteur du piéton qui marche et qui voit les arbres, les murs des fermes etc. ${ }^{63}$.

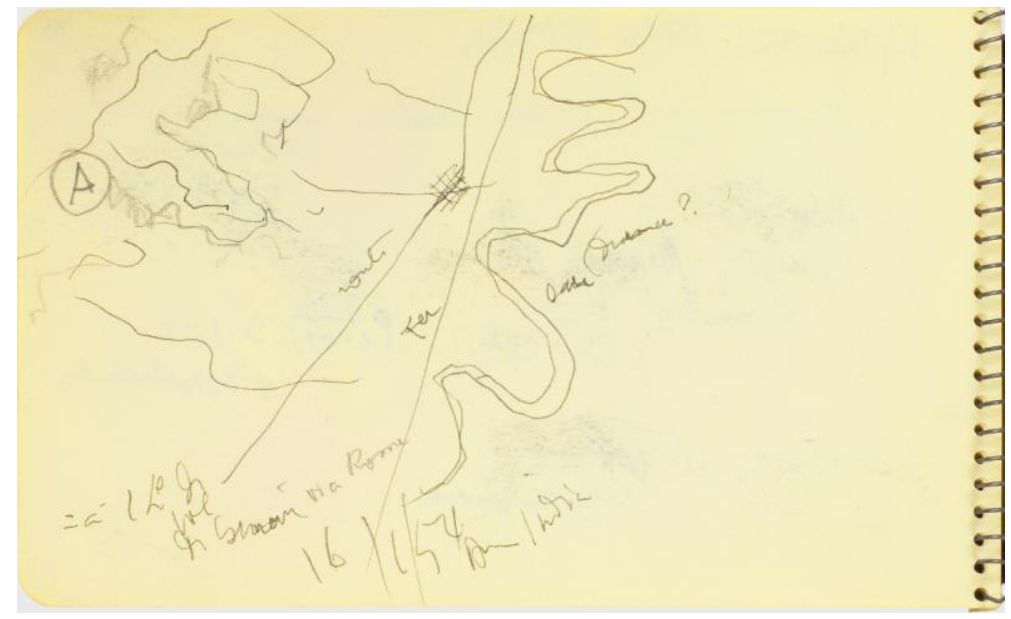

13. Les trois routes (terre, eau, fer) vues de l'avion. FLC, carnet H30, n. 1030.

Il ne s'agit pas seulement d'un changement d'échelle d'observation, mais de transformation de sa nature qui atteint presque un statut métaphysique: grâce à l'avion "nous réalisons, dans le réel, ce qui jusqu'ici n'avait été qu'une vue de l'esprit ${ }^{364}$. Les croquis de Le Corbusier saisissant la morphologie des pays survolés, se composent parfois de quelques lignes seulement: des traits qui représentent de façon dépouillée et concise les estuaires, les méandres des fleuves, les différentes voies de communication - les trois routes: terre, eau, fer - qui s'alignent parallèlement etc. ${ }^{65}$. Des images graphiques, presque abstraites, capables néanmoins de fournir "une leçon de hydrographie, de physique" ${ }^{, 66}$. Entre la petite échelle des grains de sable déplacés par la mer et photographiés en 1936 par Le Corbusier sur la plage du Piquey ${ }^{67}$ et celle des grandes érosions, des méandres infinis et des estuaires des villes de l'Afrique du nord ou de l'Amérique du sud, la différence ne réside que dans la dimension des objets représentés. Le processus naturel d'évaporation et condensation de l'eau qui régit la planète entière comparée depuis l'avion à un œuf poché6 ${ }^{68}-$, devient pour Le Corbusier l'une des questions essentielles sur laquelle réfléchir, peu importe l'échelle de ses effets.

\footnotetext{
${ }^{62}$ FLC, carnet P60, n. 501.

${ }^{63}$ FLC, carnet S67, n. 928.

${ }^{64}$ De Pierrefeu, François et Le Corbusier: La maison des hommes. Paris: Plon, 1942, p.141.

${ }^{65}$ FLC, carnet H30, n. 1030 ou encore carnet P60, n. 503.

${ }^{66}$ FLC, carnet J38, n. 439.

${ }^{67}$ Cf. Benton, Tim: LC Foto... op. cit.

${ }^{68}$ Le Corbusier: Précisions... op. cit., p. 5 : "Comme l'œuf poché, la terre est saturée d'eau en surface”.
} 


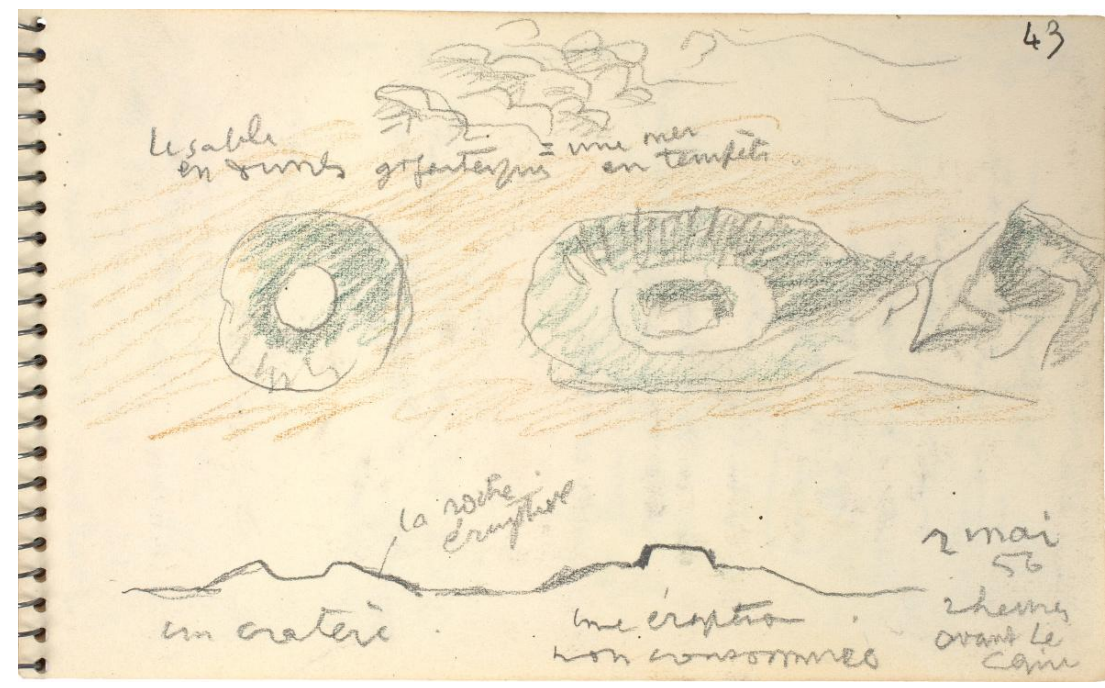

14. Sable, dunes, roches depuis l'avion, 1956. FLC, carnet K42, n. 634.

La vision aérienne ainsi que la vue plongeante qui se pose sur quelques centimètres carrés de sable, permettent de découvrir l'un des mystères de notre terre qui, avec ses ondulations, ses roches et ses petits ou grands mouvements, est capable de provoquer en nous "la notion de sublime" $"$. Pas étonnant alors que dans un dessin de 1933 - année pendant laquelle il survole avec Louis Durafour le désert allant d'Alger à Laghouat et Ghardaïa - Le Corbusier note cette petite équation: “avion=philosophie/spectacle à thèse" ${ }^{\text {"70 }}$. Ce moyen de transport a la capacité de soulever des questions et des problèmes essentiels concernant le cadre de vie des hommes et la vue sans fin qu'il autorise d'un "horizon sans rupture limite du disque terrestre",71 accorde à Le Corbusier une sérénité et une fusion avec le cosmos: "Je suis dans un état de parfait équilibre, de parfaite sérénité, presque effusion, à $6000 \mathrm{~m}$ d'altitude...."72.

\section{Les cartes postales entre panoramas urbains et territoriaux}

On ne connait pas très bien l'emploi que Le Corbusier faisait des cartes postales, en dehors du lien avec son œuvre picturale ou de leur intégration dans ses textes publiés ${ }^{73}$. D'après un examen de la collection, il parait toutefois que les vues panoramiques depuis les hauteurs ainsi que celles depuis les avions occupent une part importante du fonds concernant l'architecture et l'espace urbain. D'une part elles ont probablement servi d'“aide-mémoire" au sein d'un processus d'apprentissage et de connaissance, d'autre part elles semblent être le miroir parfait de sa conscience qu'une nouvelle échelle de lecture du territoire terrestre va désormais orienter la vision humaine. C'est peut-être pour travailler sur cette nouvelle perception de la ville autorisée par l'aviation, que Le Corbusier achète des cartes postales reproduisant l'île de Manhattan ou San Francisco photographiées depuis le ciel, mais jamais à une hauteur trop importante, afin de pouvoir saisir la ville dans son contexte naturel (montagne, mer etc.) ainsi que ses reliefs. La vue aérienne du grand carrefour Slussen à Stockholm réalisé dans les années 1930, fait partie de la collection ${ }^{74}$. L'infrastructure est photographiée depuis un angle à même de saisir les différents niveaux routiers entrelacés, ainsi que la contiguïté au centre urbain. Les espaces liés au monde du transport suscitent chez le Corbusier depuis toujours une attention spéciale. Cette carte postale rappelle en effet

\footnotetext{
${ }^{69}$ Ibid., p. 15.

${ }^{70}$ FLC, cahier de dessins, n. 5, 1933, p. 19.

${ }^{71}$ FLC, carnet J39, n. 448

${ }^{72}$ Le Corbusier: Carnets 2...op. cit., n. 934.

${ }^{73}$ Cf. Burriel Bileza, Luis: Le Corbusier. La passion des cartes. Bruxelles: Mardaga, 2013, p. 22.

${ }^{74}$ FLC, cartes postales, L5-9-18.
} 
les différents clichés photographiques pris par l'architecte au cours de l'un de ses voyages en avion à Anvers, dans lesquelles il avait fixé le port et ses alentours ${ }^{75}$.

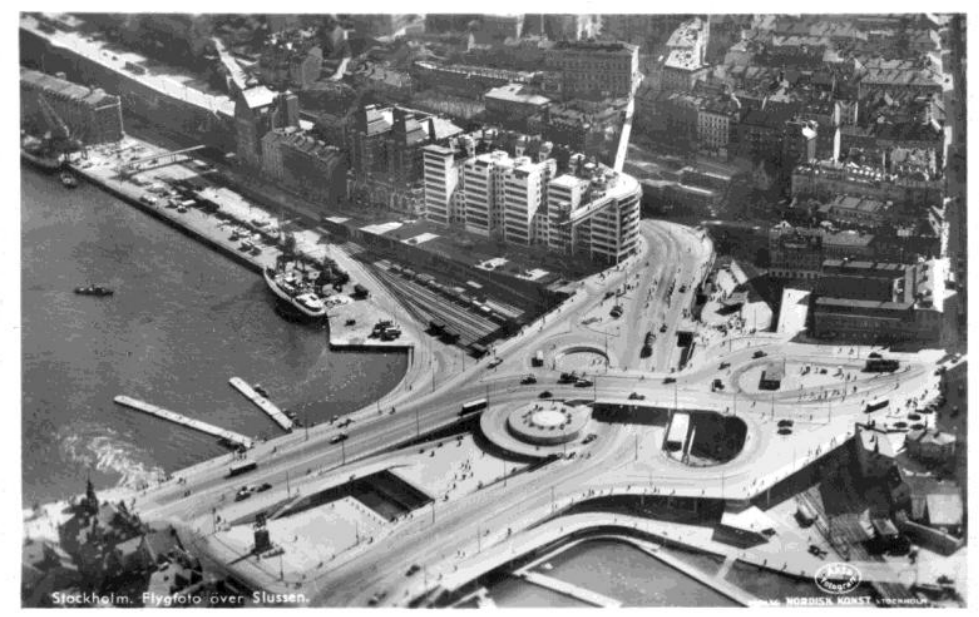

15. Carte postale représentant une vue aérienne du grand carrefour Slussen de Stockholm. FLC, L5-9-18.

Mêmes les cartes postales des remparts d'Aigues Mortes (cliché de la Compagnie Aérienne Française) ou des denses tissus urbains de Venise offrent des vues plongeantes très réelles et peu abstraites ${ }^{76}$. La présence de quelques vues aériennes nocturnes (Rio de Janeiro, New York etc.) témoigne également de l'intérêt de Le Corbusier pour l'effet que l'éclairage électrique pourrait susciter auprès d'un voyageur découvrant ces agglomérations. Dans ses notes prises au cours de ses voyages en avion il a aussi souvent fait allusion à la vision nocturne des villes et au rôle des lumières électriques dans l'appréhension de la grille urbaine, de la géométrie du tracé (à Téhéran par exemple) aussi bien que dans la révélation de 1" "affiche de bienvenu" des villes, comme lorsqu'il affirme, en 1960, que "l'arrivée de nuit sur Bombay est saisissante" avec ses bords de mer "ceinturés... de lumière" ". Nombreuses sont aussi les cartes postales conservées dans cet immense fonds iconographique, reproduisant le skyline des villes européennes ${ }^{78}$.

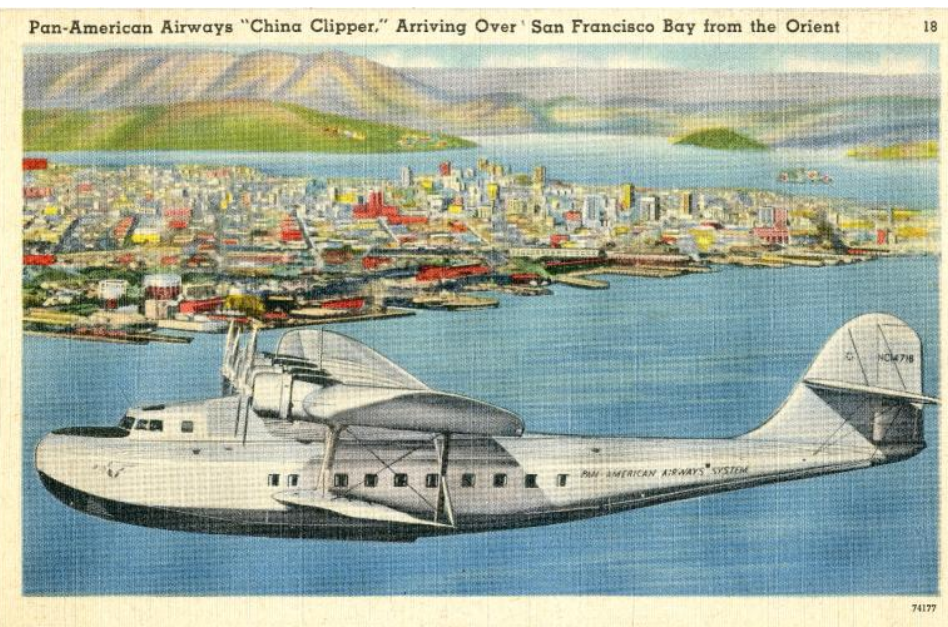

16. Carte postale avec une vue aérienne de San Francisco. FLC, L5-4-80.

\footnotetext{
${ }^{75}$ FLC, L5-2-39 e L5-2-40

${ }^{76}$ FLC, L5-5-6; L5-5-215, L5-8-287.

${ }^{77}$ FLC, carnet P60, n. 500.

${ }^{78}$ Cf. Burriel Bileza, Luis: Le Corbusier... op. cit.
} 
Le choix de cadrage de ces cartes postales qui comprennent aussi une vue de New York depuis le Hudson River, est en parfaite résonance avec les silhouettes dessinées ou photographiées par l'architecte - parfois depuis un bateau - depuis le temps de ses voyages de jeunesse. Par ailleurs, le format de ses carnets de croquis, employés à l'horizontale, ressemble étrangement à celui des cartes postales. Deux moyens de figuration qui témoignent du même intérêt pour l'effet que la silhouette d'une ville peut provoquer sur le touriste/spectateur/acheteur. Qu'il s'agisse de photos prises par lui-même, d'annotations ou de cartes postales achetées, Le Corbusier ne privilégie jamais les images représentant les centres urbains depuis la verticale, mais des panoramas visant à donner le premier aperçu de la ville qu'on s'apprête à découvrir. Un aperçu visuel que l'architecte a toujours considéré comme important, et qui devient un véritable leit motiv à partir de ses voyages dans les des années Trente.

\section{De la vision humaine à $1,60 \mathrm{~m}$ à celles infernales des trains et des automobiles}

“...il n'est, avec l'avion, que le paquebot en mer et le pied du marcheur sur la route, qui permettent ce qu'on pourrait appeler des visions humaines: on voit, l'œil transmet calmement" ${ }^{\prime 79}$. Le danger des eaux, par exemple, que le colon qui habite en Uruguay ne comprend pas, peut devenir clair seulement grâce à la vue depuis l'avion. Mais c'est la vue à 1,60 m de hauteur qui vient confirmer et compléter les premières impressions engendrées par l'arrivée par bateau ou par aéroplane. En effet, l'œil du piéton qui a "conservé des jambes et des yeux à 1,60 m $d u$ sol" saisit les traces naturelles de manière assez différente de l'avion qui "a gagné des ailes" ${ }^{\text {" }}$ : si la vue des prairies, des fleuves, des forêts vierges confère au marcheur des sensations de "noblesse, d'exubérance, d'opulence, de vie", la même vue, depuis le ciel, n'apparait que de la "moisissure"81.

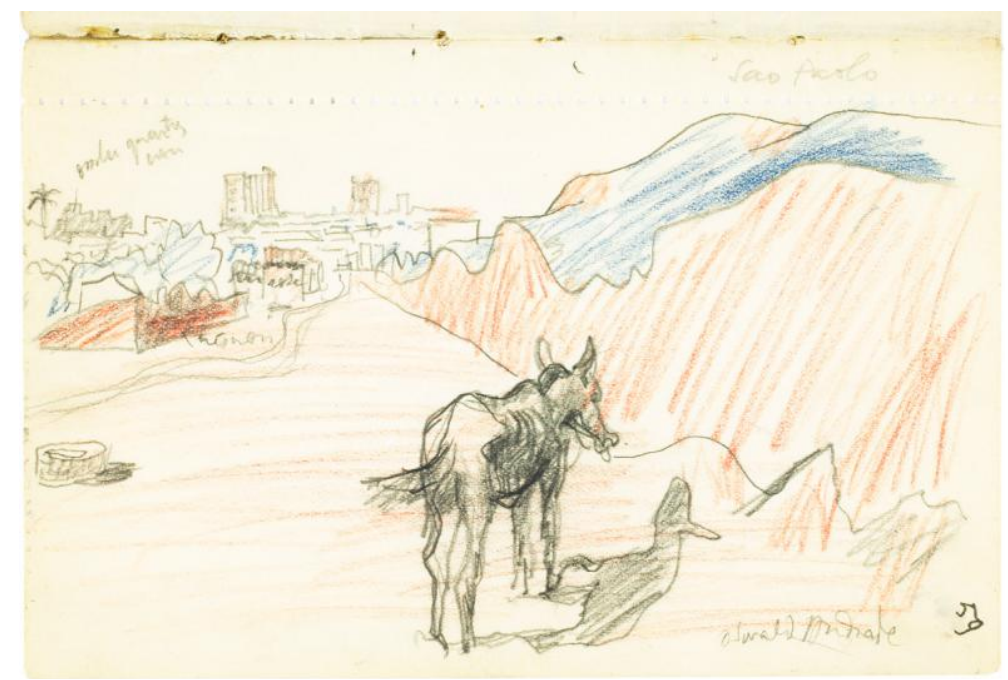

17. Vue de Sao Paulo depuis la route. FLC, carnet B4, n. 260.

La promenade en ville prévoit généralement de monter sur les sommets pour scruter le tissu urbain depuis les hauteurs et souvent du point de vue opposé à celui offert par la vision depuis la baie. "Du haut des «Favellas» on voit toujours la mer, les rades, les ports, les îles, l'océan, les montagnes, les estuaires; le nègre voit tout cela...; une fierté est dans l'œil du nègre qui voit tout ça; l'œil de l'homme qui voit de vastes horizons est plus hautain, les vastes horizons confèrent de la dignité; c'est une réflexion d'urbaniste..." ${ }^{„ 22}$. Parallèlement, les pieds du marcheur permettent aussi de pénétrer dans le cœur des villes et de découvrir les différentes solutions

\footnotetext{
${ }^{79}$ Le Corbusier: Précisions... op. cit., p. 8.

${ }^{80}$ FLC, carnet F26, n. 846.

${ }^{81}$ Le Corbusier: Précisions... op. cit., p. 7.

${ }^{82}$ Ibid, p. 235.
} 
de l'habitat historique et/ou moderne. À Buenos Aires, “j'ai parcouru à pied nombre de rues... J'ai regardé, $v u$ et compris..." $" 83$. Le dessin de Sao Paulo depuis la route témoigne de la vision calme et paisible que la vitesse du pas humain ou animal autorise: Le Corbusier a le temps de dessiner aussi, à côté du paysage, l'âne qui l'accompagne ${ }^{84}$.

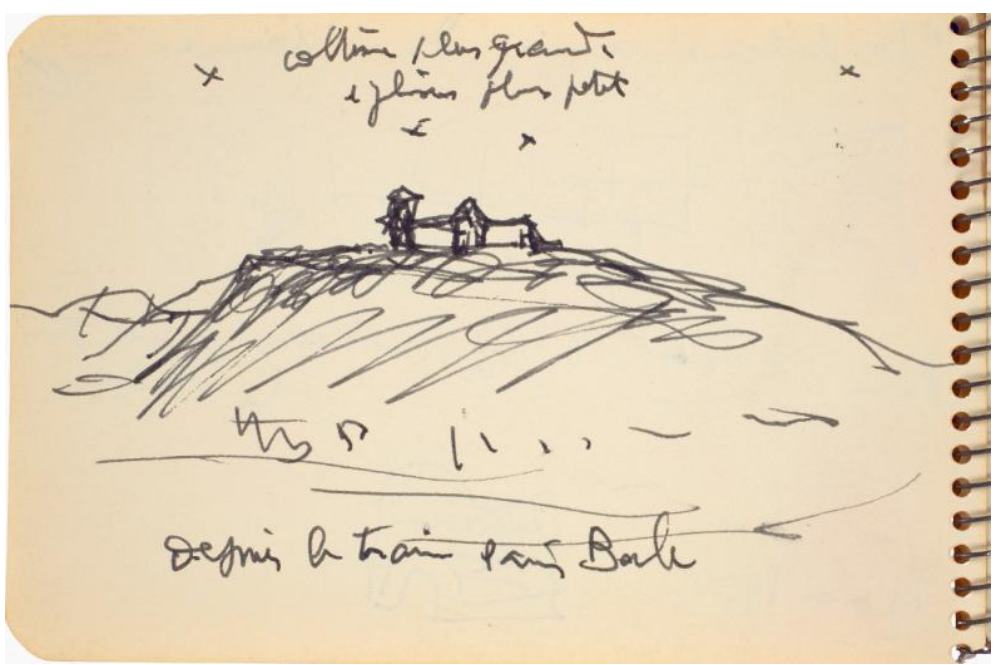

18. Esquisse de Ronchamp depuis le train, 1950. FLC, carnet D17, n. 272.

À Alger, l'architecte se balade dans la Casbah; à Rio de Janeiro il esquisse les maisons et signale leur ancrage au sol ainsi que les magnifiques panoramas visibles par leurs fenêtres ${ }^{85}$. À ces visions humaines, Le Corbusier oppose celles “inhumaines et infernales ... offertes d'un train ou d'une auto, même d'une bécane"86. L'architecte ne semble pas tellement aimer les chemins de fer. D'une part car ils ont remplacé la traditionnelle entrée en ville qui se faisait "en pompe étincelante par le pont-levis et les portes des rois (porte Saint-Denis, porte Saint-Martin, seules demeurées) et la sente millénaire jusqu'au cour de la cité, où sont les cathédrales et les hôtels de ville" ${ }^{87}$ par "la lèpre qui s'étale autour du cliquetis du train sur les aiguillages" ${ }^{88}$. D'autre part, car les chemins de fer ont déversé en ville une foule épouvantable, en contribuant à la rendre invivable. Mais c'est aussi la vitesse de ce transport qui est mise en cause, puisqu'elle a supplanté le rythme lent et naturel des êtres vivants et de leurs moyens de transport, sans pour autant être à même d'entrainer une nouvelle façon d'appréhender la ville. Les premières esquisses de Ronchamp élaborées en mai 1950 “depuis train Paris-Bâle" ${ }^{\text {} 89}$ constituent les rares exemples de saisie/insertion d'une architecture au milieu de son contexte paysager pris depuis ce moyen de transport.

Mais la pensée de Le Corbusier semble parfois hésiter et se brouiller lorsqu'il analyse les quatre routes (automobile, avion, bateau, chemin de fer) et les visions qu'elles ont entrainées. Si la rapidité symbolise la civilisation machiniste et a fait "considérer d'un oxil nouveau la planète" ${ }^{\text {, }}$, celle qui appartient proprement aux trains est considérée toutefois par l'architecte comme une "vitesse... [qui] brouille le paysage", alors que celle des avions échappe à cette critique, car leur "course foudroyante" donnerait "la sensation étrange de la presque immobilité" ${ }^{\prime \prime 1}$. Les diagrammes réalisés lors de la sixième conférence tenue à Buenos Aires en 1929

\footnotetext{
${ }^{83}$ Le Corbusier: Précisions... op. cit., p. 35.

${ }^{84}$ FLC, carnet B4, n. 260.

${ }^{85}$ Le Corbusier: Précisions... op. cit., p. 10.

${ }^{86}$ Le Corbusier: Ibid, p. 8.

${ }^{87}$ Le Corbusier: Sur les 4 ... op. cit., p. 113.

${ }^{88}$ Ibid, pp. 113-114.

${ }^{89}$ Le Corbusier: Carnets 2...op. cit., n.272-273.

${ }^{90}$ Le Corbusier: Sur les 4 ... op. cit., p. 40.

${ }^{91}$ Ibid, pp. 41-42.
} 
dans lesquels Le Corbusier esquisse la courbe de la vitesse qui, depuis 1850, a augmenté de façon exponentielle grâce aux "chemins de fer, paquebots, avions, dirigeables, automobiles T.S.F., téléphone",92, mérite notre attention.

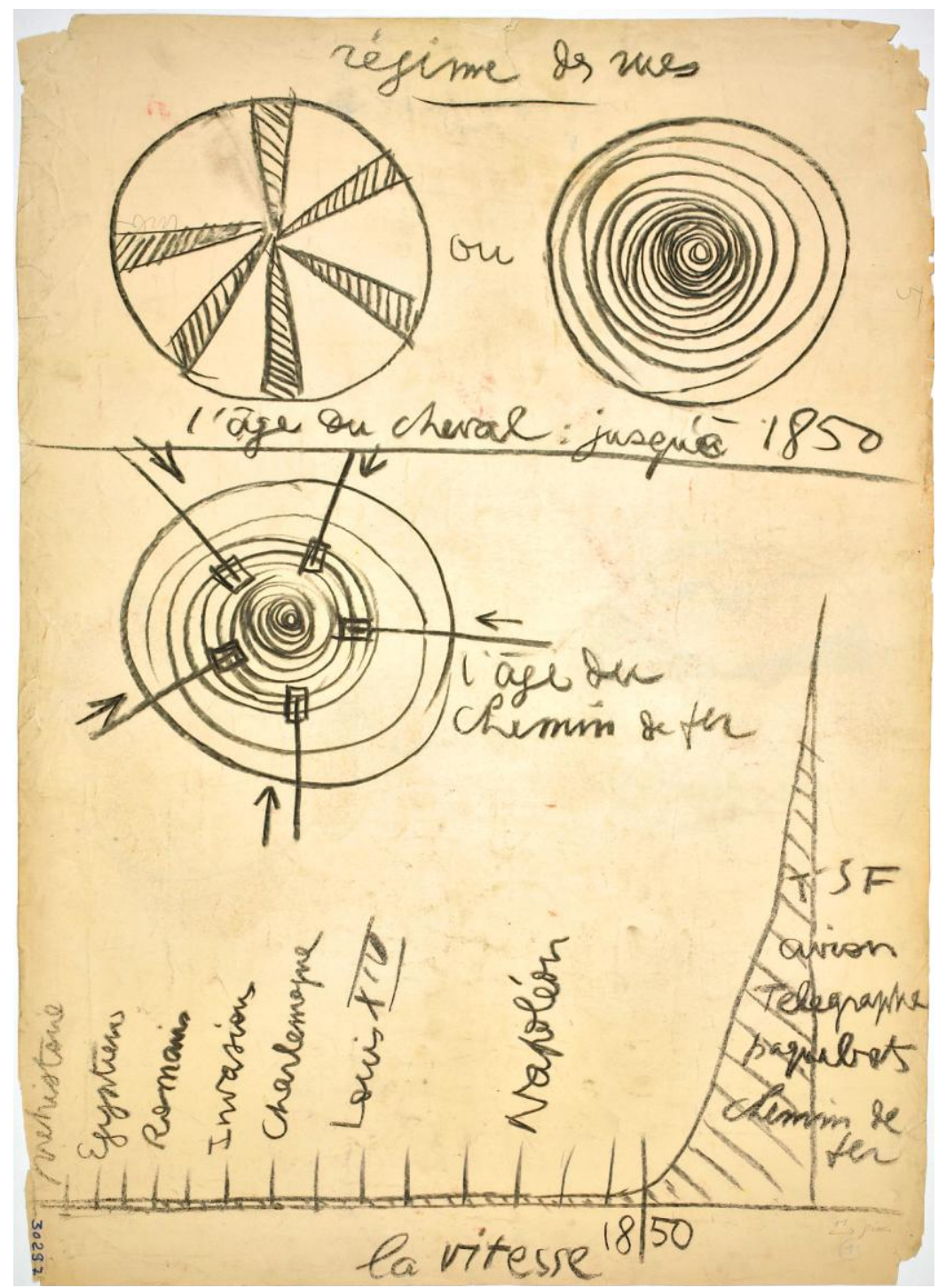

19. Diagramme de la vitesse. Esquisse élaborée lors de la sixième conférence tenue à Buenos Aires en 1929. FLC plan 30297A.

La conception de la route moderne comme axe de circulation très rapide est certainement à l'origine de la définition de vision inhumaine et infernale, élaborée par le Corbusier en 1930. La célérité, dont le but est celui de faire baisser les encombrements, autorise une vision cinétique qui s'accommode peu à un enregistrement fidèle de la réalité. L'automobile peut seulement aider à la perception de la topographie du terrain parcouru: "en auto, nous avons fait des expériences: celle par exemple du temps considérable qu'il faut pour aller d'un point à un autre: vallonnements, contours, pentes, etc." ${ }^{, 3}$. Ou, dans le meilleur des cas, elle permet à Le Corbusier de se faire une idée sommaire de la construction des maisons et du mode de l'habitat, comme lorsqu'il voyage en 1951 sur sa jeep de Chandigarh à New Dehli ${ }^{94}$.

\footnotetext{
92 Cf. Benton, Tim: Le Corbusier conférencier. Paris. Editions Le Moniteur, 2007, pp. 184-185. Un des deux croquis a été publié dans Le Corbusier: Précisions... op. cit., p. 148.

${ }_{93}^{93}$ Le Corbusier: Précisions... op. cit, p. 240.

${ }^{94}$ FLC, carnet E18, 343-346.
} 


\section{Conclusion}

Pour Le Corbusier, voir - et ne pas simplement regarder - depuis un bateau ou un avion, entraine une approche cognitive et interprétative de la ville et de ses caractères urbains et paysagers que nul autre moyen de transport n'autorise. Il s'agit de visions (avec la vue à 1,60 m de hauteur) qui trouvent dans la complémentarité, le couronnement de leurs objectifs. La grande distance depuis l'objet autoriserait, selon l'architecte, une perception davantage globale, précise et objective, tout en permettant de prendre des décisions rapides dans le domaine de la conception du projet architectural et urbain. En particulier, l'aéroplane est désormais devenu la métaphore d'un regard nouveau. La prédilection pour une vision lente et presque "immobile" consentie par un paquebot ou un avion révèle une démarche accordant à la première découverte d'un site - silhouette, trame, morphologie etc. une importance sans précédent. Mais l'approche essentiellement visuelle que l'on peut avoir depuis une embarcation arrivant à proximité de la côte, trouve son véritable aboutissement grâce à la contemplation depuis le ciel. "The bird's eye view"95 est désormais capable non seulement de déclencher une nouvelle échelle d'appréhension du monde, des sensations inédites, mais aussi d'engendrer une nouvelle philosophie de vie.

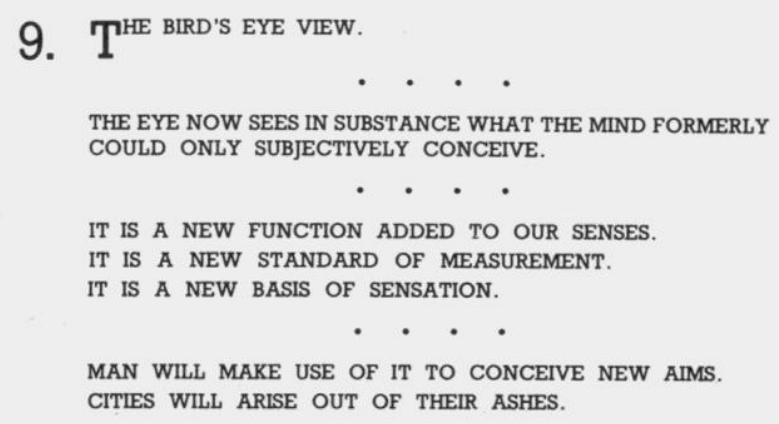

20. Extrait de Aircraft, 1935. Le Corbusier: Aircraft. London: The Studio, 1935, p. 96.

\section{Liste des images}

1. La forteresse de Smederevo. Photographie 1911. FLC, L5-1-124.

2. Photo prise lors du voyage dans les Balkans en 1911. FLC, L4-20-170.

3. Panorama d'Istanbul, 1911. FLC, 2383.

4. Séquence sténographique de Manhattan. Le Corbusier: Quand les cathédrales étaient blanches. Voyage au pays des timides. Paris: Plon, 1937, pp. 315-322.

5. Vue de Barcelone depuis la mer, 1932. FLC, carnet C10, n. 636.

6. Un des premiers croquis pour Rio de Janeiro. FLC, dessin 5033.

7. Rio de Janeiro, vue depuis l'avion avec croquis de son projet pour la ville. FLC, A3.18.98.

8. Feuille esquissant le projet de rédiger un chapitre d'Urbanisme sur la vue d'avion FLC, B2-3-80.

9. Le Corbusier lors d'un voyage en avion. FLC, L5-2.41.

10. Vue aérienne d'une ville du désert, 1933. FLC, dessin 5010.

11. Paysage algérien depuis l'avion, 1933. Le Corbusier: Aircraft. London: The Studio, 1935, p. 116.

\footnotetext{
${ }^{95}$ Le Corbusier: Aircraft, op. cit., p. 96.
} 
12. Depuis l'avion, Le Corbusier note les couleurs des territoires survolés, 1958. FLC, carnet J37, n. 334.

13. Les trois routes (terre, eau, fer) vues de l'avion. FLC, carnet H30, n. 1030.

14. Sable, dunes, roches depuis l'avion, 1956. FLC, carnet K42, n. 634.

15. Carte postale représentant une vue aérienne du grand carrefour Slussen de Stockholm. FLC, L5-9-18.

16. Carte postale avec une vue aérienne de San Francisco. FLC, L5-4-80.

17. Vue de Sao Paulo depuis la route. FLC, carnet B4, n. 260.

18. Esquisse de Ronchamp depuis le train, 1950. FLC, carnet D17, n. 272.

19. Diagramme de la vitesse. Esquisse élaborée lors de la sixième conférence tenue à Buenos Aires en 1929. FLC, plan 30297A.

20. Extrait de Aircraft, 1935. Le Corbusier: Aircraft. London: The Studio, 1935, p. 96.

\section{Bibliographie}

A.A.V.V.: L'invention d'un architecte. Le voyage en Orient de Le Corbusier. Paris: Editions de la Villette, 2013.

Benton, Tim: "Le Corbusier photographe secret". In Herschdorfer, Nathalie et Umstätter, Lada (Dir.): Construire l'image. Le Corbusier et la photographie. Paris: Textuel, 2012, pp. 30-53.

Benton, Tim: LC Foto: Le Corbusier Secret Photographer. Zürich: Lars Müller, 2013.

Benton, Tim: Le Corbusier conférencier. Paris. Éditions Le Moniteur, 2007.

Bonillo, Jean-Lucien (Dir.): Le Corbusier. Visions d'Alger. Paris: Éditions de la Villette, 2012.

Burriel Bileza, Luis: Le Corbusier. La passion des cartes. Bruxelles: Mardaga, 2013.

Casabella. $\mathrm{N}^{\circ}$ 531-532. Janvier-Février 1987.

De Pierrefeu, François et Le Corbusier: La maison des hommes. Paris: Plon, 1942.

Duboy, Philippe: Le Corbusier. Croquis de voyages et études. Paris: La Quinzaine Littéraire-Louis Vuitton, 2009.

Gresleri, Giuliano: "Dal diario al progetto. I Carnet 1-6 di Le Corbusier". In Lotus. N.68. 1991, pp. 6-21.

Le Corbusier: Aircraft. London: The Studio, 1935.

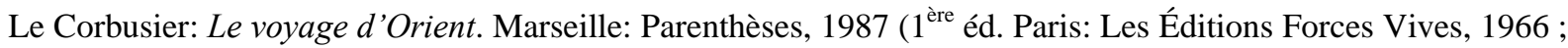
éd. it. Il viaggio d'Oriente. Faenza: Faenza Editrice, 1974).

Le Corbusier: Précisions sur un état présent de l'architecture et de l'urbanisme. Paris: G. Crès et Cie. Coll. "L'esprit nouveau", 1930.

Le Corbusier: Sur les 4 routes. Paris: Denoël, 1970 (1 $1^{\text {ère }}$ éd. 1941).

Le Corbusier: Vers une architecture. Paris: Arthaud, 1977 (1 $1^{\text {ère }}$ éd. 1923).

Le Corbusier. Aventures photographiques. Paris, Éditions de la Villette, 2014.

Le Corbusier: Quand les cathédrales étaient blanches. Voyage au pays des timides. Paris: Plon, 1937 (éd. ital. Quando le cattedrali erano bianche. Faenza: Faenza editrice, 1975).

Lemonnier, Aurélien: "Des yeux qui accusent: Le Corbusier et la ville vue d'avion". In Lampe Angela (Dir): Vues d'en haut. Metz: Centre Pompidou, 2013, pp. 258-261.

Mazza, Barbara: Le Corbusier et la fotografia. La vérité blanche. Firenze: Firenze University Press, 2002. 